\title{
Subretinal Human Umbilical Tissue-Derived Cell Transplantation Preserves Retinal Synaptic Connectivity and Attenuates Müller Glial Reactivity
}

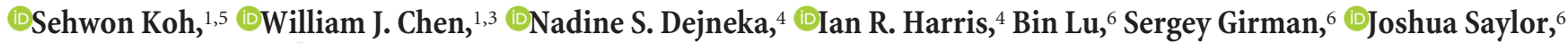 \\ Shaomei Wang, ${ }^{6}$ and ${ }^{\circledR C}$ Cagla Eroglu ${ }^{1,2,3,5}$

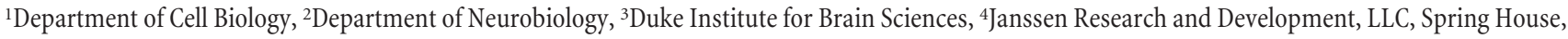 \\ Pennsylvania 19477, ${ }^{5}$ Regeneration Next, Duke University Medical Center, Durham, North Carolina 27710, and ${ }^{6}$ Department of Biomedical Sciences, \\ Regenerative Medicine Institute, Cedars-Sinai Medical Center, Los Angeles, California 90048
}

Human umbilical tissue-derived cells (hUTC or palucorcel) are currently under clinical investigation for the treatment of geographic atrophy, a late stage of macular degeneration, but how hUTC transplantation mediates vision recovery is not fully elucidated. Subretinal administration of hUTC preserves visual function in the Royal College of Surgeons (RCS) rat, a genetic model of retinal degeneration caused by Mertk loss of function. hUTC secrete synaptogenic and neurotrophic factors that improve the health and connectivity of the neural retina. Therefore, we investigated the progression of synapse and photoreceptor loss and whether hUTC treatment preserves photoreceptors and synaptic connectivity in the RCS rats of both sexes. We found that RCS retinas display significant deficits in synaptic development already by postnatal day 21 (P21), before the onset of photoreceptor degeneration. Subretinal transplantation of hUTC at P21 is necessary to rescue visual function in RCS rats, and the therapeutic effect is enhanced with repeated injections. Synaptic development defects occurred concurrently with morphological changes in Müller glia, the major perisynaptic glia in the retina. hUTC transplantation strongly diminished Müller glia reactivity and specifically protected the $\alpha 2 \delta$-1-containing retinal synapses, which are responsive to thrombospondin family synaptogenic proteins secreted by Müller glia. Müller glial reactivity and reduced synaptogenesis observed in RCS retinas could be recapitulated by CRISPR/Cas9-mediated loss-of-Mertk in Müller glia in wild-type rats. Together, our results show that hUTC transplantation supports the health of retina at least in part by preserving the functions of Müller glial cells, revealing a previously unknown aspect of hUTC transplantation-based therapy.

Key words: cell transplantation; MERTK; Müller glia; retinal degeneration; synapse formation; thrombospondins

\section{Significance Statement}

Despite the promising effects observed in clinical trials and preclinical studies, how subretinal human umbilical tissue-derived cell (hUTC) transplantation mediates vision improvements is not fully known. Using a rat model of retinal degeneration, the RCS rat (lacking Mertk), here we provide evidence that hUTC transplantation protects visual function and health by protecting photoreceptors and preserving retinal synaptic connectivity. Furthermore, we find that loss of Mertk function only in Müller glia is sufficient to impair synaptic development and cause activation of Müller glia. hUTC transplantation strongly attenuates the reactivity of Müller glia in RCS rats. These findings highlight novel cellular and molecular mechanisms within the neural retina, which underlie disease mechanisms and pinpoint Müller glia as a novel cellular target for hUTC transplantation.

\section{Introduction}

Age-related macular degeneration is the leading cause of blindness in individuals over the age of 60 (Lim et al., 2012). Currently,

\footnotetext{
Received June 2, 2017; revised Jan. 22, 2018; accepted Jan. 26, 2018.

Author contributions: S.K., N.S.D., I.R.H., and C.E. designed research; S.K., W.J.C., N.S.D., B.L., S.G., J.S., and S.W. performed research; I.R.H. and C.E. contributed unpublished reagents/analytic tools; S.K., W.J.C., N.S.D., S.G., and S.W. analyzed data; S.K., W.J.C., N.S.D., and C.E. wrote the paper.

This work was supported in part by National Institutes of Health Grants NEI 1F32 EY027997 and NIA 2T32AG000029 to S.K., Duke University Chancellor's Discovery Award and Kahn Neurotechnology Development
}

there are no effective treatments available for most of these patients, particularly those afflicted with geographic atrophy, the

grant to C.E., and Duke Regeneration Next Initiative Postdoctoral Fellowship to S.K. We thank Bin Gao (Janssen R\&D) for statistical support.

Parts of the work described were performed under sponsored research agreements between Duke University and Janssen R\&D, and Cedars-Sinai Medical Center and Janssen R\&D.N.S.D. and I.R.H. are employees of Janssen R\&D. The remaining authors declare no competing financial interests.

Correspondence should be addressed to Dr. Cagla Eroglu, Duke University Medical Center, Durham, NC 27710. E-mail: c.eroglu@cellbio.duke.edu.

DOI:10.1523/JNEUROSCI.1532-17.2018

Copyright $\odot 2018$ the authors $\quad 0270-6474 / 18 / 382923-21 \$ 15.00 / 0$ 
advanced form of dry age-related macular degeneration, characterized by degeneration of the retinal pigment epithelium (RPE), choriocapillaris, and death of photoreceptors (PRs) (Bird et al., 2014).

Multiple cell-based therapies are currently under clinical investigation for the treatment of geographic atrophy and fall into two categories: (1) cell replacement strategies that focus on replacing dysfunctional RPE cells; and (2) cell preservation strategies in which transplanted cells provide trophic support to promote multiple aspects of retinal health, including neuronal survival and connectivity (Klassen, 2016; Venugopalan et al., 2016; Zhao et al., 2017). Human umbilical tissue-derived cells (hUTC; palucorcel), harvested from postpartum umbilical cord, that is void of cord blood, are thought to use the latter strategy (Lund et al., 2007). These cells have been successfully tested in a Phase $1 / 2 \mathrm{a}$, multicenter, open-label, dose escalation, safety study (Ho et al., 2017). A Phase $2 b$, multicenter, double-masked, randomized study is ongoing to evaluate the safety and clinical response to the subretinal administration of hUTC (www.ClinicalTrials.gov, Identifier NCT02659098).

The therapeutic potential of hUTC administration has also been demonstrated in various animal disease models of stroke (Zhang et al., 2011, 2012, 2013; Jiang et al., 2012; Moore et al., 2013) and in a rat model of retinal degeneration, the Royal College of Surgeons (RCS) rat, which has a loss-of-function mutation in the Mertk gene (Lund et al., 2007). Previous studies, including our recent report, have provided strong evidence that paracrine factors secreted by hUTC enhance functional recovery and protect neurons from progressive degeneration and rescue RPE phagocytic dysfunction (Alder et al., 2012; Koh et al., 2015; Cao et al., 2016).

The factors secreted by hUTC include growth factors and cytokines with general neuroprotective effects. Moreover, hUTC enhance synaptic connectivity and promote neuronal outgrowth by secreting thrombospondin (TSP) family proteins, TSP1, TSP2, and TSP4 (Koh et al., 2015). TSPs induce synaptogenesis by their interaction with their neuronal receptor, the gabapentin receptor $\alpha 2 \delta$-1 (Eroglu et al., 2009). TSPs and $\alpha 2 \delta$ - 1 are also present in adult rodent retina; however, the cell source that provides TSPs in the retina has not been confirmed (Huang et al., 2013; Yafai et al., 2014). Furthermore, the involvement of impaired TSP/ $\alpha 2 \delta$ - 1 signaling and abnormal synaptic development in the diseased retina has not been explored.

In the brain, astrocytes secrete TSPs to control excitatory synapse formation between neurons (Christopherson et al., 2005). However, retinal astrocytes reside outside of the synaptic layers. Instead, a specialized radial glial cell type, Müller glia (MG), spans the entire neural retina and extends their processes into the synaptic plexiform layers. MG are required for maintenance of $\mathrm{PR}$ health and connectivity (Williams et al., 2010); however, molecular mechanisms that underlie MG-neuron interactions are understudied.

In this study, we focused on the effects of hUTC on neural retina and investigated the cellular mechanisms that underlie how subretinal hUTC transplantation rescues vision in the RCS rat. We found that the RCS rat displayed significant deficits in synaptic development and these deficits strongly correlated with early morphological and reactivity changes in the MG and reduced expression of MG-secreted TSP1 and TSP2 and their neuronal receptor, $\alpha 2 \delta-1$. hUTC transplantation significantly preserved PRs and protected synaptic connectivity. In particular, the multiple hUTC injection paradigm, which provided best visual recovery, also attenuated the reactive changes in MG and
Table 1. Rat groups and subretinal hUTC treatments used in this study

\begin{tabular}{llllll}
\hline Group & Animals & Treatment & Day of treatment & Total cells/eye & Injection volume \\
\hline 1 & Long Evans & None & - & - & - \\
2 & RCS & None & - & - & - \\
3 & RCS & hUTC & P22 & 20,000 & $2 \mu \mathrm{l}$ \\
4 & RCS & BSS & P21 & - & $2 \mu \mathrm{l}$ \\
5 & RCS & hUTC & P60 & 20,000 & $2 \mu \mathrm{l}$ \\
6 & RCS & hUTC & P21 and P60 & 20,000 (P21) & $2 \mu \mathrm{l}(\mathrm{P} 21)$ \\
& & & & 20,000 (P60) & $2 \mu \mathrm{l}(\mathrm{P60})$ \\
\hline
\end{tabular}

specifically protected the $\alpha 2 \delta$-1-containing synapses. Furthermore, we found that loss-of-Mertk function only in MG of wildtype (WT) rats is sufficient to impair synaptic development and cause activation of MG, similar to what is observed in the RCS rats. Our findings highlight novel cellular and molecular mechanisms within the neural retina, which underlie disease mechanisms and pinpoint $\mathrm{MG}$ as an important cellular target for treatment of retinal degeneration.

\section{Materials and Methods}

Animal welfare statement. All animal procedures were conducted according to the ARVO Statement for the Use of Animals in Ophthalmic and Vision Research and in accordance with the institutional animal care and use committee guidelines: Institutional Animal Care and Use Committee Protocol Numbers A-173-14-07, A147-17-06 (Duke University) and Institutional Animal Care and Use Committee 003801 (Cedars-Sinai Medical Center).

hUTC preparation for subretinal injection. hUTC were isolated and cryopreserved as previously described (Koh et al., 2015). Cryopreserved hUTC ( $\sim 31.3$ population doublings; $2 \times 10^{6}$ viable cells $/ \mathrm{ml}$ ) were used for the present study. On each day of injection, frozen cells ( 2 or 3 vials) were thawed at $37^{\circ} \mathrm{C}$ in a water bath for $\sim 2 \mathrm{~min}$. Upon thaw, cells were transferred to a single $15 \mathrm{ml}$ conical tube containing $8 \mathrm{ml}$ of balanced saline solution (BSS) Sterile Irrigating Solution (Alcon). An additional 1 $\mathrm{ml}$ of BSS was added to the cryovials, and the rinse was subsequently transferred to the $15 \mathrm{ml}$ conical tube. Cells were centrifuged at $250 \times g$ for $5 \mathrm{~min}$ at room temperature. The supernatant was removed, and the pellet was resuspended in $5 \mathrm{ml}$ BSS. Cells were counted using a C-Chip Neubauer Improved Disposable Hemocytometer (IN CYTO) to determine the total number of viable cells. Remaining cells were subsequently centrifuged for $5 \mathrm{~min}$ at $250 \times \mathrm{g}$. The supernatant was removed, and the cells were resuspended to a final concentration of $\sim 10,000$ cells $/ \mu$ l in BSS. Each cell suspension was transferred from the conical tube to an Eppendorf tube and placed on ice. The time that cells were placed on ice was recorded. This time was used to set a $2 \mathrm{~h}$ window to complete the subretinal injections.

Isolation and culture of primary rat retinal ganglion cells (RGCs). RGCs were purified by sequential immunopanning from P7 (postnatal day 7) Sprague Dawley rat retinas (Charles River) as previously described (Winzeler and Wang, 2013). Briefly, retinas were dissected and dissociated with papain (6 U/ml, Worthington). Dissociated cells were panned first with Bandeiraea Simplicifolia Lectin I (Vector Laboratories) coated Petri dishes to remove immune cells, cell debris, and fibroblasts. The unbound cells were transferred to a Petri dish coated with anti-Thy1 (clone T11D7) antibody for specific isolation of RGCs. The purified RGCs were gently trypsinized and replated onto poly-D-lysine and laminin-coated glass coverslips in 24-well plates (35,000 cells/coverslip). RGCs were cultured in a serum-free growth medium containing B27 (Invitrogen), BDNF, CNTF, insulin, and forskolin (full recipe of the media can be found in Winzeler and Wang, 2013).

Experimental design for subretinal hUTC injection. Pigmented female and male dystrophic RCS rats (postnatal day P21-P22, P60) were used for the study. Age-matched Long-Evans (LE) rats served as controls. Animals were divided into 6 study groups, with 6 study animals per group (Table 1). All animals were maintained at Cedars-Sinai Medical Center. 
Subretinal injections. Subretinal injections were performed in RCS rats on P21-P22 (Groups 3 and 4) and P60 (Group 5). Group 6 animals received 2 injections in the same eye. The first injection was administered on P21 and the second injection on P60. All injections were performed in the right eye. The left eyes were not treated. Animals were anesthetized intraperitoneally with $75 \mathrm{mg} / \mathrm{kg}$ zetamine (VetOne) and $0.25 \mathrm{mg} / \mathrm{kg} \mathrm{dex}$ medetomidine (Zoetis) diluted in bacteriostatic $0.9 \% \mathrm{NaCl}$ (Hospira). The eye was dilated with $1 \%$ tropicamide ophthalmic solution USP (Bausch \& Lomb) followed by $2.5 \%$ phenylephrine hydrochloride ophthalmic solution (Paragon, BioTek). The eye was stabilized using a nonabsorbable suture (4-0) (Ethicon). The suture was placed behind the equator of the eyeball to pull the eyeball forward and allow for exposure of the dorsal-temporal portion of the eye.

To observe the fundus clearly, Gonak (Hub Pharmaceuticals) was placed on the cornea of the globe. A plastic ring was subsequently placed on the eyelid to keep the Gonak in place. A scissor was used to cut away conjunctiva, and a $301 / 2 \mathrm{G}$ metal needle was used to make a sclerotomy at upper temporal region of the eye; $2 \mu \mathrm{l}$ cell suspension was drawn into a sterile glass pipette (internal diameter $50-150 \mu \mathrm{m}$ ) via a plastic tube filled with BSS that was attached to a $25 \mu \mathrm{l}$ Hamilton syringe. To reduce intraocular pressure and to limit the efflux of cells, the cornea was punctured using a $30 \mathrm{l} / 2 \mathrm{G}$ metal needle. Cells or BSS ( $2 \mu$ l volume) were injected through the site of the sclerotomy. Immediately after injection, the fundus was examined for retinal damage or signs of vascular distress. The wound was sutured with a nonabsorbable surgical suture (10-0) (Ethicon). The suture around the eyeball was removed, and then the eyelid was put into its normal position. Finally, $0.5 \%$ erythromycin ophthalmic ointment (Bausch \& Lomb) was used locally. Rats were given $1 \mathrm{mg} / \mathrm{kg}$ atipamezole (Orion) intraperitoneally to reverse the effects of the dexmedetomidine. The animals recovered from anesthesia on warm pads $\left(37^{\circ} \mathrm{C}\right)$ before they were returned to their holding room. Animals that received hUTC and BSS injections received daily dexamethasone (Fresenius Kabi) injections $(1.6 \mathrm{mg} / \mathrm{kg}$, i.p.) for 2 weeks following the subretinal procedure. Additionally, these animals received cyclosporine-A (Teva Pharmaceuticals) in their drinking water $(210 \mathrm{mg} / \mathrm{L})$ throughout the course of the entire experiment.

Visual function assessments. All animals were tested for spatial visual acuity at different predetermined time points (P30/31, P60, and P88P93) using an optomotor testing apparatus (Cerebral Mechanics) as previously described (Prusky et al., 2004; Lund et al., 2007). Optokinetic response (OKR) allowed for noninvasive gross measures of visual acuity as a function of reflexive image stabilization.

Luminance threshold (LT) recordings were performed on 3 animals from each group on P90-P95, as previously described (Girman et al., 2005). Recordings were made from both the treated and untreated eyes. Briefly, animals were anesthetized, a small skin incision was made over the superior colliculi (SC), and 15-20 openings were drilled through the skull over the area of the SC dorsal projection. Glass-coated tungsten microelectrodes (resistance: $0.5 \mathrm{~m} \Omega$; bandpass $500 \mathrm{~Hz}$ to $5 \mathrm{kHz}$ ) were introduced through the openings into the SC. The brightness of a $5^{\circ}$ spot was varied using neutral density filters (minimum steps of $0.1 \mathrm{log}$ unit) over a baseline level of $5.2 \mathrm{log}$ units until a response double the background activity was obtained: this was defined as the threshold level for that point on the visual field. A total of 15 positions were recorded from each SC. Data were expressed as a graph of percentage of the SC area with a LT below defined levels.

Retina preparation for immunohistochemistry (IHC). After visual function assessments, the retinas from LE and RCS rats were collected. Animals were terminated on P94-P96 by $\mathrm{CO}_{2}$ asphyxiation, followed by bilateral pneumothorax. Eyes were removed and immersed in 2\% PFA for $1 \mathrm{~h}$ and subsequently infiltrated with $10 \%, 20 \%$, and $30 \%$ sucrose. Eyes were maintained in each solution for $1 \mathrm{~h}$ at room temperature and then transferred to $4^{\circ} \mathrm{C}$ overnight in $30 \%$ sucrose. Eyes were embedded in OCT (frozen tissue matrix) and cut in sequence (10 $\mu \mathrm{m}$ horizontal sections apart) on a cryostat. Every sixth section was placed on the same slide as the first section, and a total of four sections (50 $\mu \mathrm{m}$ apart) were collected per slide. A total 40-50 slides/eye were cut.

For the IHC analyses during development, age-matched LE and RCS retinas (P14, P21, and P30) were collected by intracardially perfusing with TBS ( $25 \mathrm{~mm}$ Tris-base, $135 \mathrm{~mm} \mathrm{NaCl}, 3 \mathrm{~mm} \mathrm{KCl,} \mathrm{pH} \mathrm{7.6)} \mathrm{supple-}$ mented with $7.5 \mu \mathrm{M}$ heparin followed with $4 \%$ PFA (Electron Microscopy Sciences) in TBS. The eyes are enucleated and lens was removed by making an incision in the cornea. The eyecups were fixed with $4 \%$ PFA in TBS for $2 \mathrm{~h}$ at room temperature. The eyecups were cryoprotected with $30 \%$ sucrose in TBS overnight and were then embedded in OCT (TissueTek) compound and frozen.

Identification of synapses by immunocytochemistry. Retina sections were washed three times and then permeabilized in PBS with $0.4 \%$ Triton X-100 (PBST; Roche) at room temperature. Sections were blocked in 5\% NGS, normal donkey serum, or BSA in PBST for $1 \mathrm{~h}$ at room temperature. Primary antibodies (mouse anti-Bassoon 1:500 [RRID:AB_10618753, ADIVAM-PS003-F, Enzo], rabbit anti-mGluR6 1:150 [RRID:not applicable, RA13105, Neuromics], guinea pig anti-VGlut1 1:750 [AB5905, Millipore], rabbit anti-PSD95 1:500 [RRID:AB_87705, 51-6900, Invitrogen], mouse anti-Gephyrin 1:250 [RRID:AB_1279448, 147-021, Synaptic Systems], goat anti-TSP1 1:200 [RRID:AB_2201958, AF3074, R\&D Systems], goat-anti-TSP2 1:200 [RRID:AB_2202068, AF1635, R\&D Systems], mouse anti-glutamine synthetase 1:1000 [RRID:AB_397879, 610517, BD Biosciences], rabbit anti-SOX9 1:4000 [RRID:AB_2239761, AB5535, Millipore], goat anti-ChAT[RRID:AB_11214092, AB144P, Millipore], rabbit anti- $\alpha 2 \delta$-1 [RRID:AB_258885, C5105, Sigma-Aldrich]), rabbit anti- $\alpha 2 \delta$-1 [RRID:AB_2039785, ACC-015, Alomone Labs], and rabbit anti-GFAP [RRID:AB_10013382, Z033429-2, Dako] were diluted in 5\% NGS or 5\% BSA containing PBST. Sections were incubated overnight at $4^{\circ} \mathrm{C}$ with primary antibodies. For TSP staining, the primary antibodies were incubated for $48 \mathrm{~h}$ at $4^{\circ} \mathrm{C}$ as previously described (Huang et al., 2013). Secondary Alexa-fluorophore-conjugated antibodies (Invitrogen) were added (1:200 in PBST with 5\% NGS or 5\% BSA) for $2 \mathrm{~h}$ at room temperature. Slides were mounted in Vectashield with DAPI (Vector Laboratories), and images were acquired on a Leica SP5 and SP8 confocal laser-scanning microscopy.

Quantification of synapses (synapse analysis). Three or four animals per age of LE or RCS were used for synapse analysis. Three independent retina sections per each group of treatments (Groups 1-6) or age (P14, $\mathrm{P} 21$, and P30) were used for IHC. The 5- $\mu$ m-thick confocal $z$ stacks (optical section depth $0.33 \mu \mathrm{m}, 15$ sections $/ z$ stack) of the retinas were imaged at $63 \times$ magnification on a Leica SP5 confocal laser-scanning microscope. Maximum projections of three consecutive optical sections (corresponding to $1 \mu \mathrm{m}$ total depth) were generated from the original $z$ stack. Analyses were performed blind as to strain and/or treatments. The Puncta Analyzer plugin (written by Barry Wark, available upon request from C.E.; c.eroglu@cellbio.duke.edu) for ImageJ 1.29 (National Institutes of Health; http://imagej.nih.gov/ij/; version ImageJ 1.29 is available at https://sites.duke.edu/eroglulab/quantification-of-synapses/) was used to count the number of colocalized puncta. This quantification method is based on the fact that presynaptic and postsynaptic proteins (e.g., VGluT1 and PSD95) are not within the same cellular compartments of neurons (axons vs dendrites, respectively) and would only appear to partially colocalized at synaptic junctions due to their close proximity. This quantification method yields an accurate estimation of the number of synapses both in vitro and in vivo because it measures colocalization as opposed to staining of a single presynaptic or postsynaptic protein because synaptic proteins also often accumulate in extrasynaptic regions during the course of their life cycle. In agreement, numerous previous studies by us and others showed that synaptic changes observed by this quantification method is verified by techniques such as EM and electrophysiology (Christopherson et al., 2005; Eroglu et al., 2009; Kucukdereli et al., 2011; Allen et al., 2012; Risher et al., 2014; Koh et al., 2015; Singh et al., 2016). Details of the quantification method have been described previously (Ippolito and Eroglu, 2010). Briefly, 1- $\mu \mathrm{m}$-thick maximum projections are separated into red and green channels, backgrounds are subtracted (rolling ball radius $=50$ ), and thresholds are determined to detect discrete puncta without introducing noise. Minimum pixel size of puncta was set as 4 to remove any background noise. The Puncta Analyzer plugin then uses an algorithm to detect the number of puncta that are in close proximity across the two channels, yielding quantified colocalized puncta. To calculate percentage of WT colocalization, colocalized 
puncta values for WT were averaged, then all image values (WT and $\mathrm{KO}$ ) were converted to percentage of the calculated WT average.

TUNEL assay. To detect degenerative PRs, apoptotic cells were detected by TUNEL (In Situ Cell Death Detection Kit, Roche) staining according to the manufacturer's protocol. Briefly, cryosectioned (10-12 $\mu \mathrm{m})$ retina was washed with PBS for $30 \mathrm{~min}$ and permeabilized in $0.1 \%$ Triton X-100 for $2 \mathrm{~min}$ on ice. The slides were washed twice with PBS followed by incubation with TUNEL reaction mixture for 6 min at $37^{\circ} \mathrm{C}$ in the dark. The slides were washed three times with PBS and mounted in Vectashield with DAPI (Vector Laboratories). The images of TUNELpositive cells were acquired on a Leica SP5 confocal laser-scanning microscope.

RNA FISH. A set of FISH probes targeting either rat Thbs1 or Thbs 2 was purchased from Stellaris (LGC Biosearch Technologies). Each probe set was composed of 48 oligonucleotides (20 nucleotides each) that selectively bind to transcripts of either TSP1 (Thbs1) or TSP2 (Thbs2). The probe sets were labeled with fluorescent dye CAL Fluor Red 610 or Quasar 670, for Thbs 1 or Thbs2, respectively. Briefly, $10 \mu \mathrm{m}$ retina sections were fixed with $4 \%$ PFA for 15 min and washed twice with PBS containing RNase inhibitor (Invitrogen). The sections were permeabilized with ethanol for $2 \mathrm{~h}$ at room temperature. After wash and rehydration with PBS, the sections were sequentially incubated with primary (mouse antiglutamine synthetase [GS], 1:200) and secondary (anti mouse-IgG AlexaFluor-488, 1:200) antibodies for $1 \mathrm{~h}$ at room temperature with PBS washes between steps. After immunostaining, the sections were postfixed with $4 \%$ PFA for $15 \mathrm{~min}$ at room temperature followed by a PBS wash. Then, RNA FISH was performed following the manufacturer's recommended protocol.

CRISPR/SaCas9-mediated ablation of MER receptor tyrosine kinase (MERTK), adeno-associated virus (AAV) design, virus production, and intravitreal injection. To ablate Mertk gene specifically in MG, we purchased Clustered Regularly Interspaced Short Palindromic Repeats (CRISPR)/SaCas9 vector from Addgene (pX601, Plasmid \#61591) and cloned an sgRNA (GCTGGATGTGATCTTCCAGTC) targeting rat Mertkgene between BsaI sites. AAVs containing CRISPR/SaCas9 were produced by the Duke Viral Vector Core using MG-specific capsid (ShH10) from Dr. John Flannery (University of California-Berkley) (Klimczak et al., 2009).

To verify that designed sgRNA targeted Mertk locus and disrupted MERTK expression, cortical astrocytes or retinal glia were isolated from P1 Sprague Dawley rat pups using standard methods as described by (McCarthy and de Vellis, 1980). The $2 \times 10^{6}$ cells in a $10 \mathrm{~cm}$ dish were transduced with AAV-CRISPR/SaCas9. We verified CRISPR/SaCas9mediated genome excision, first by conducting T7 endonuclease I assay (T7E1). To do so, rat genomic DNA was isolated from cortical or retinal astroglial cultures at day 3 after transduction, and $\sim 1100$ bp of genomic region around sgRNA target site was amplified by PCR using the following primers: forward, TCGTGTGGGTGAGGCAGTGTTC; and reverse, CATGCTCAAGGCTCTGGGTTCC. The PCR amplicon was then subjected to T7E1 assay to verify cleavage, and the mutations visualized by the T7E1 assay were further confirmed by sequencing.

P7-P10 LE pups were used for intravitreal injections; then, retinas were collected at P21 and P45. A total of $2 \mu \mathrm{l}$ containing $1 \times 10^{12} \mathrm{vg} / \mathrm{ml}$ of AAV-CRISPR was intravitreally injected per eye using $5 \mu$ l Hamilton syringes.

Preparation of retinal glia-conditioned media. Retinal glia were isolated from P1 Sprague Dawley pups and cultured as previously described (McCarthy and de Vellis, 1980; Trachtenberg and Packey, 1983). Retinal glia were grown in $10 \mathrm{~cm}$ tissue culture dishes (Corning) and treated with $5 \mu \mathrm{l}$ of $1 \times 10^{12} \mathrm{vg} / \mathrm{ml} \mathrm{AAV}$ viral particles, then incubated overnight. At day 5 after transduction, the retina glia were washed twice with warm DPBS (Invitrogen) and $10 \mathrm{ml}$ of conditioning media, composed of DMEM (Invitrogen) supplemented with L-glutamine ( $2 \mathrm{~mm}$, Invitrogen), sodium pyruvate (1 mM, Invitrogen), penicillin $(100 \mathrm{U} / \mathrm{ml})$, and streptomycin $(100 \mu \mathrm{g} / \mathrm{ml}$, Invitrogen), was added to each dish. Media were conditioned for $5 \mathrm{~d}$, then collected and concentrated 10 times by using 5 $\mathrm{kDa}$ molecular weight cutoff Vivaspin 20 centrifugal concentrators (Sartorius). The protein concentration of each conditioned media was measured by the standard BCA assay (Thermo Scientific) following the manufacturer's recommendations. Aliquots of retinal glia-conditioned media in low protein-binding tubes (Eppendorf) were rapidly frozen in liquid nitrogen and were stored at $-80^{\circ} \mathrm{C}$ until use.

Retinal glia $R G C$ coculture experiments. For neuron/glia coculture experiments, 150,000 retinal glial cells were seeded into transwell inserts in growth media, described above. The next day, these cells were treated with AAV-CRISPR containing either control or Mertk-targeting sgRNA. At day 5 after transduction, retina glia were treated with hUTC-conditioned medium (UCM) prepared as previously described (Koh et al., 2015) for $3 \mathrm{~d}$ before coculture. On the day of coculture, the inserts with cells were washed twice with DPBS, then medium was replaced with RGC growth media. Transwell inserts were transferred into 24 -well plates with 4 DIV RGCs that were grown on glass coverslips. The RGCs and retinal glial cells were then cocultured for $6 \mathrm{~d}$ to allow for synapses to form between cells. RGCs cultured alone (without inserts) served as a control.

Western blot analysis. Either retinal glia-conditioned media or glial cell lysates were used for Western blot analysis. The conditioned media were prepared as described above. Whole-cell lysates were prepared using RIPA lysis buffer supplemented with protease inhibitor (Sigma-Aldrich). Protein concentration was determined for each sample by standard BCA assay (Thermo Scientific), and $20 \mu \mathrm{g}$ of protein from each sample was boiled at $95^{\circ} \mathrm{C}$ for $5 \mathrm{~min}$. Proteins were separated in 10\% SDS-PAGE gels (Bio-Rad) and were then transferred onto PVDF membranes (Millipore). To detect protein expression, goat anti-TSP1 (1:250, RRID:AB_2201958), TSP2 (1:250, RRID:AB_2202068) (R\&D Systems), mouse anti-GS (1: 1000, RRID:AB_397879, BD Biosciences), goat anti-MERTK (1:250, RRID:AB_2098563, R\&D Systems), and rabbit anti-GFAP (1:2000, RRID:AB_10013382, Dako) were used. HRP-conjugated anti-goat antibody (1:5000, RRID:AB_357236, R\&D Systems) or, fluorescent anti-goat antibody (1:5000, RRID:AB_2650427, LI-COR Biosciences) was used as secondary antibody. The detection was performed with an ECL Western Blotting Analysis System kit (GE Healthcare) or Odyssey System (LICOR Biosciences).

Statistical analysis. Statistical analyses of the quantified data were performed using Student's $t$ test or one-way ANOVA followed by post hoc test (Tukey's HSD), if applicable. For luminance threshold analyses, we performed Levene's test for homogeneity of variance to confirm that the variances of different groups were the same and then we applied Tukey's HSD multiple comparisons. JMP Genomics Pro 13.0 software (SAS) was used for all statistical analysis of the data. All data were expressed as mean \pm SEM.

\section{Results}

\section{Recovery of visual function by subretinal hUTC} transplantation depends on the time of cell administration

A previous study demonstrated that subretinal transplantation of hUTC on P21 recovers visual function in the RCS rats (Lund et al., 2007). The therapeutic effects of hUTC transplantation were gained without transdifferentiation of transplanted cells into retinal cells. This observation suggested that hUTC support visual function recovery via paracrine effects that promote the health and function of the retinal cells. To achieve a better understanding of the cellular and molecular mechanisms involved in therapeutic effects of these cells on visual function, we first questioned whether it is necessary to administer hUTC to the RCS rats within a specific period of time to observe benefits. Furthermore, we also tested whether the therapeutic benefits of this treatment could be enhanced by repeated cell administration. To do so, we compared the efficacy of hUTC injection at two different time points: P21 and P60. Cryopreserved hUTC were freshly thawed and prepared on the day of transplantation at 10,000 cells/ $\mu$ l in BSS and then subretinally injected to right eyes of RCS rats on P21 (G3), P60 (G5), or both at P21 and P60 (G6) (Fig. 1A). Left eyes did not receive any treatment. All rats were under immunosuppression to eliminate the effects of cell transplantation-driven immune response to the experimental outcomes. Age-matched LE (G1), RCS rats receiving no treatment (G2), and RCS rats receiving 

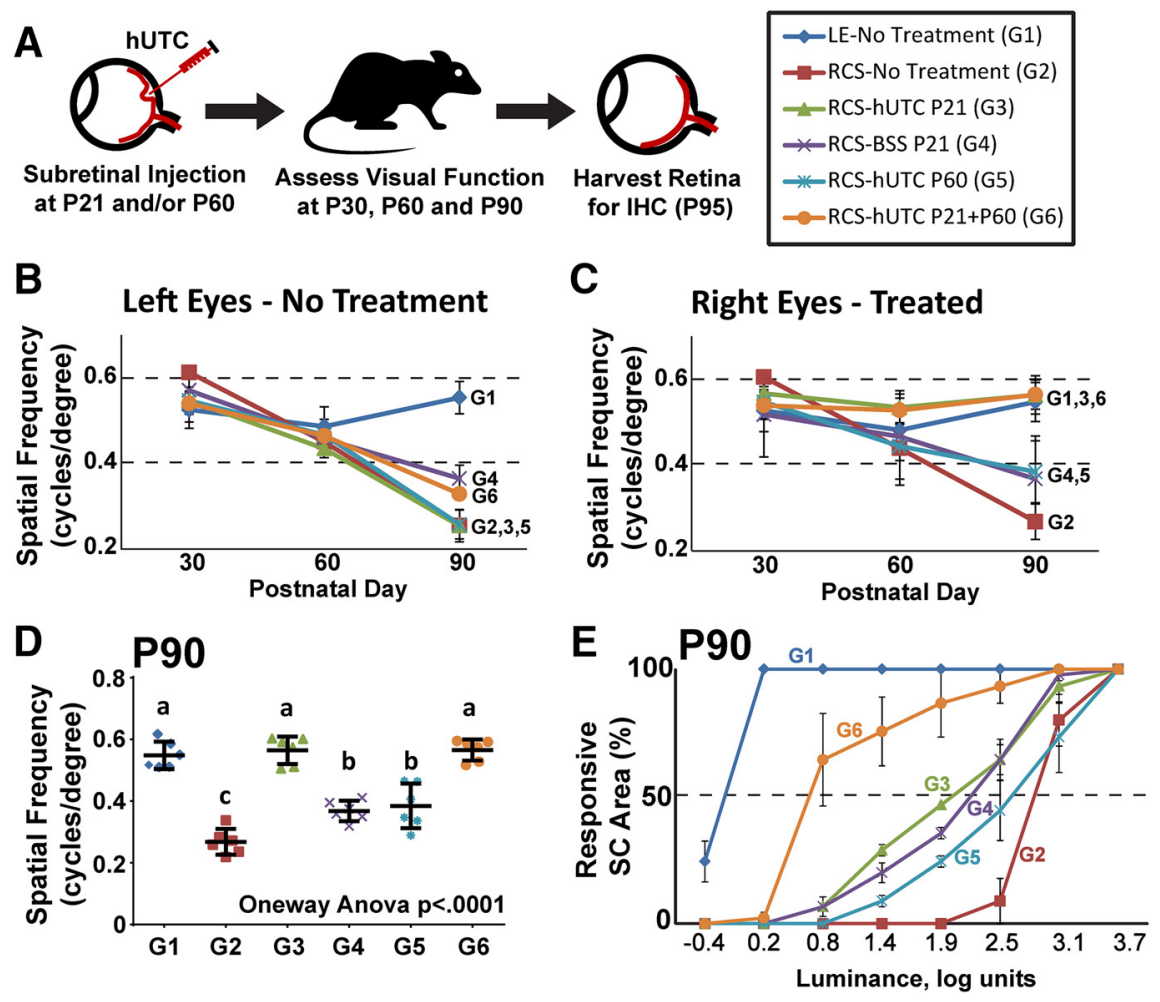

Figure 1. Recovery of visual function by subretinal hUTC transplantation depends on cell injection on P21. $\boldsymbol{A}$, Schematic representation of the experimental design. hUTC were injected into the subretinal space on P21 and/or P60. Visual function recovery was assessed on $\mathrm{P} 30, \mathrm{P} 60$, and $\mathrm{P} 90-\mathrm{P} 95$, and then retina samples were harvested from the same animals on $P 95$. $\boldsymbol{B}, 0$ KR tests demonstrated that left eyes without injection showed progressive loss of vision in RCS rats. $C$, Right eyes of RCS rats that received hUTC subretinally on P21 alone (G3) or on P21 and P60 (G6) demonstrated vision responses that were comparable with healthy control LE rats (G1), whereas the vehicle control group (BSS, G4) or P60 hUTC-treated group (G5) progressively lost visual function similar to that of untreated controls (G2).D, OKR results of right eyes on P90 demonstrate that $\mathrm{G} 3$ and $\mathrm{G} 6$ had significantly improved visual function. $p<0.05$ (one-way ANOVA and Tukey's post hoc test). Different letters are statistically different. $\boldsymbol{E}$, LTR on P90 - P95 demonstrated that the superior colliculus of $\mathrm{G} 6$ animals was significantly more responsive to light stimuli than animals in G3. Data are mean \pm SEM. OKR tests were performed in all animals in each group $(n=6)$. Luminance recordings were performed on 3 animals in each group.

vehicle (i.e., BSS only, G4) served as controls. Visual function was assessed by measuring the OKR on P30, P60 and P90 followed by luminance threshold response (LTR) on P90-P95. Following LTR testing, the retinas were collected for IHC analyses.

OKR testing did not reveal any significant differences in visual function among the 6 study groups on P30 or P60; however, by P90 untreated RCS rats (G2), the vehicle-injected group (G4) and those RCS rats treated with cells on P60 (G5) showed significant vision loss. On the other hand, RCS rats receiving subretinal hUTC transplantation once on P21 (G3) or twice on P21 and P60 (G6) showed optokinetic responses that were equivalent to healthy LE rats (Fig. $1 C, D$; one-way $\operatorname{ANOVA}\left(F_{(5,66)}=16.7772, p<0.0001\right)$ and Tukey's post hoc test). The left eyes (without treatment) from all RCS animals displayed impaired visual function at P90 (Fig. 1B).

To evaluate the effects of hUTC transplantation on retinal synaptic function, we measured the retinal response to light stimuli by recording luminance thresholds (LTRs) from the superior colliculus, a brain region that receives direct synaptic inputs from the RGCs. LTRs were performed as previously described (Girman et al., 2005). Interestingly, the retinas of G6 (repeated injections at P21 and P60) demonstrated significantly lower luminance thresholds and broader area under the curve than G3 (single injection at P21, Tukey's HSD, $p=0.003$ ) and G4 (vehicleinjected P21, Tukey's HSD $p=0.002$ ) at all the luminance levels (Fig. 1E).
Collectively, these results demonstrate that it is critical to inject hUTC at an early stage of retinal degeneration to maximize vision preservation in RCS rats, and the therapeutic effects of hUTC transplantation are enhanced by repeated injections.

hUTC treatment prevents PR apoptosis and delays outer nuclear layer (ONL) degeneration

Why is it critical to inject hUTC at P21 to have a therapeutic effect? We hypothesized that in this model it is necessary to conduct hUTC transplantation before significant PR cell loss occurs. The progressive PR loss in RCS rats has been extensively characterized with PR death first detected as early as P22 (Dowling and Sidman, 1962). Few TUNEL-positive "apoptotic" cells appear at P20, and notable TUNEL-positive PR cell staining is observed by P25 (Tso et al., 1994).

To determine the detailed timeline of retinal degeneration in this rat model, we collected and quantitatively analyzed RCS and LE retinas from untreated animals at different time points between P14 (early postnatal development, shortly after eyeopening) and P90 (late stages of the degenerative process). As previously noted, occasional apoptotic PR nuclei are present at P21 in the RCS rats, but thickness of the ONL, where the PRs reside, does not significantly differ from that of LE control rats at this time (Fig. 2A). On the other hand, we found a significant loss of ONL thickness and extensive TUNEL-positive PRs in RCS rat retinas at P30 compared with LE retinas (Fig. $2 B, D$; Student's $t$ test, $p<0.0001$ ). The majority of the PRs are lost in RCS rats by P60 (Fig. 2C,D; Student's $t$ test, $p<0.0001)$. These results show that significant PR apoptosis and loss starts to occur between P21 and P30 in the RCS retinas. We next investigated how subretinal hUTC administration affects the PR cell layer thickness and TUNEL positivity at P95. We found that hUTC injection on P21 (G3) or repeated administrations on P21 and P60 (G6) both delay $\mathrm{PR}$ loss as demonstrated by a significant increase in ONL thickness compared with the vehicle-injected RCS group (G4 on P95 (Fig. 2 E, F; one-way ANOVA, $p<0.0001$ ). However, significant PR loss still occurs in these rats compared with WT LE controls at P95 (Fig. 2 E, F; one-way ANOVA, $p<0.0001$ ). Notably, many of the remaining PRs in the vehicle-injected RCS rat retinas are TUNEL-positive; however, hUTC transplantation significantly reduces the density of TUNEL-positive cells (Fig. 2E, G; one-way ANOVA, $p<0.0001$ ). Repeated administration of hUTC at P21 and P60 further diminishes TUNEL positivity in the PR layer (Fig. 2G; one-way ANOVA, $p<0.0001$ ). Together, these results show that hUTC administration at P21 is critical for preserving PRs in the RCS rat. These results also indicate that preservation of visual function triggered by hUTC administration is at least in part due to protection of PR numbers and health. 

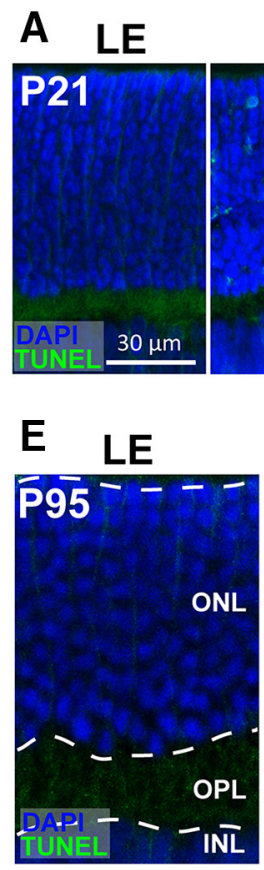

RCS
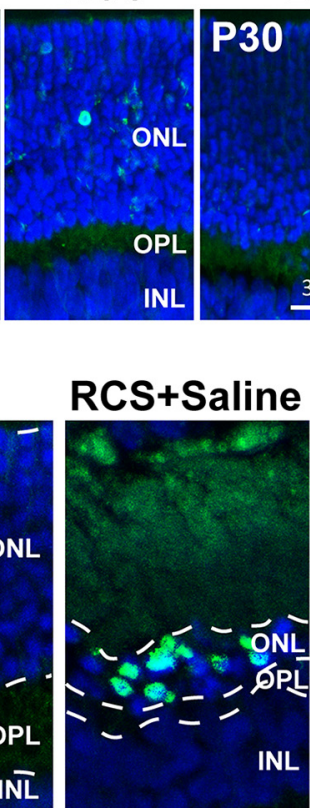

B LE

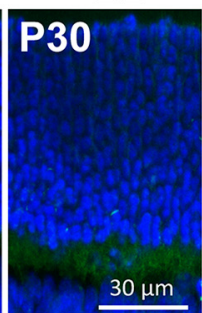

RCS

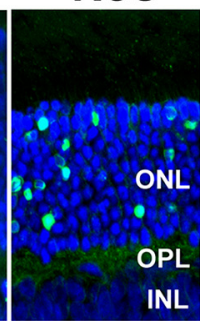

RCS+hUTC
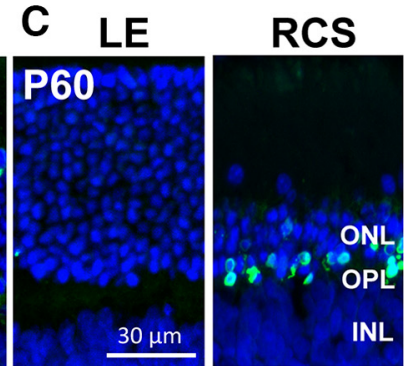

INL

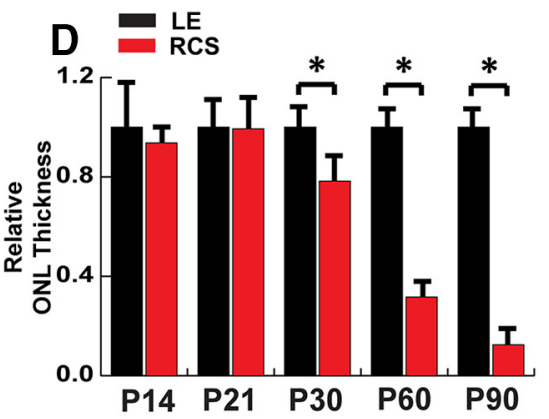

\section{P21\&60}
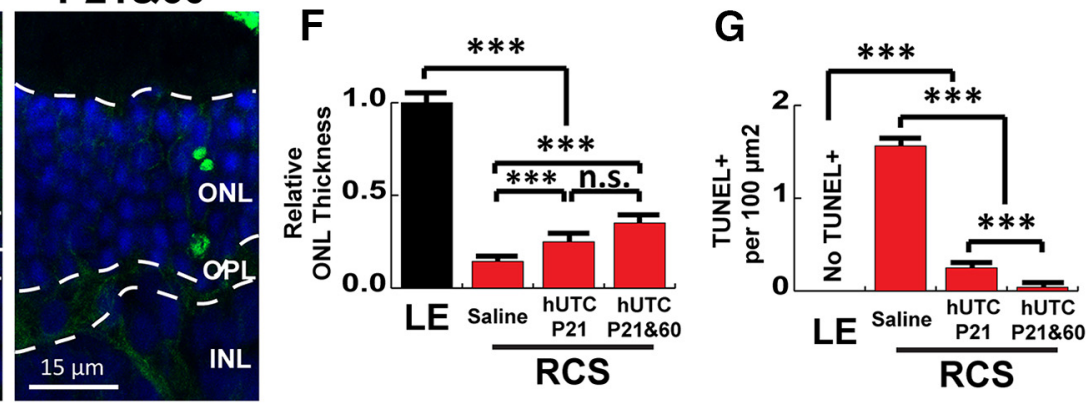

Figure 2. RCS PR loss begins between P21 and P30, and P21 hUTC injection preserves RCS PRs. Representative images of retinal sections stained with TUNEL (green) revealing apoptotic PRs in DAPI-counterstained (blue) sections at (A) P21, (B) P30, and (C) P60. D, Quantitative analysis of relative 0NL thickness of RCS normalized to age-matched controls (LE) showed significant PR loss between P21 and P30.E, Subretinal hUTC injection delayed PR loss in the RCS rat as demonstrated by increased ONL thickness and decreased TUNEL-positive PRs (green) in RCS + hUTC P21 and P60 compared with RCS + BSS.F, Quantification of the relative change in ONL thickness. G, Quantification of TUNEL-positive PR density in ONL. Data were obtained from a minimum of 3 animals of either sex and expressed as mean \pm SEM. ${ }^{*} p<0.05,{ }^{* * *} p<0.0001$.

Deficits in synapse development precede retinal degeneration in the RCS rat

Synaptic dysfunction and synapse loss are known to accompany retinal degeneration in the RCS rats (Gründer et al., 2001; Peng et al., 2003; Cuenca et al., 2005). However, it is not yet clear whether synapses are formed normally in these rats and then are lost during the degenerative process, or whether synapse development is already impaired in RCS retinas. Furthermore, previous findings suggest that the therapeutic effects of hUTC are mediated via paracrine effects (Lund et al., 2007), and hUTC-secreted factors enhance synaptic connectivity in vitro (Koh et al., 2015). Therefore, next we tested whether synaptic development is normal or impaired in the RCS rat retinas. To do so, we quantitatively analyzed the number of synapses in the RCS retinas compared with age-matched WT LE controls at P14 (before PR loss), P21 (onset of PR death), and P30 (after significant PR death has already occurred).

The neural retina is a well-organized structure with three distinct cellular layers, namely, the ONL, inner nuclear layer (INL), and the ganglion cell layer, respectively). Neurons that reside in each of these layers are connected with each other through stereotypical synaptic contacts located within the synaptic zones known as outer and inner plexiform layers (OPL and IPL, respectively) (Fig. 3A). To determine the number of synapses formed in these layers, we visualized and quantified the synapses by the colocalization of synapse type-specific presynaptic (green) and postsynaptic (red, excitatory; blue, inhibitory) markers using a previously standardized method (Ippolito and Eroglu, 2010). This method takes advantage of the fact that presynaptic and postsynaptic proteins are not in same cellular compartments (axons and dendrites, respectively), but they come in close proximity at synapses, and thus appear to colocalize when imaged at the resolution level of light microscopy.
In the OPL, the PRs form excitatory ribbon synapses onto the bipolar interneurons. We analyzed the number of OPL ribbon synapses by quantifying the colocalization of presynaptic Bassoon (green) and postsynaptic mGluR6 (red) puncta in age-matched LE and RCS retinas (Fig. 3B). We found that the number of OPL ribbon synapses in RCS retinas is significantly reduced compared with age-matched LE rats at all the time points analyzed (i.e., P14, P21, and P30; Fig. 3C; Student's $t$ test, $p=0.0084$ ). This result shows that ribbon synapse development in the OPL of RCS rats is already diminished at P14 and P21, before significant PR death occurs.

Vision-driven synaptic signals from PRs are relayed by bipolar cells of the INL. Bipolar cells project their axons into the IPL to provide presynaptic excitatory inputs onto the dendrites of RGCs (Fig. 3A). To determine whether the development of IPL bipolar cell synapses between bipolar cells and RGCs is also altered in the RCS rats, we marked these synapses by using vesicular glutamate transporter 1 (VGluT1, green) and postsynaptic density protein 95 (PSD-95, red) as presynaptic and postsynaptic markers, respectively (Fig. 3D). We found that similar to OPL, the IPL bipolar cell synapse development is also impaired in the RCS rat (Fig. $3 E$; P21 $p=0.0024$ and P30 $p<0.0001$, Student's $t$ test). Interestingly, in the LE retinas, the density of bipolar cell synapses increases dramatically between P14 and P21, whereas in the RCS rat retinas this rapid synaptic growth does not take place (Fig. $3 E$ ). Together, these results show that both OPL and IPL excitatory synapse development is negatively affected in the RCS rats. These results also show that impairments in synaptic development precede significant PR loss, indicating a possible involvement of synaptic dysfunction in the onset of retinal degeneration.

In addition to VGluT1-positive bipolar cell synapses, IPL hosts a large number of other synaptic connections. Therefore, we further characterized the deficits of synaptic development in 
A
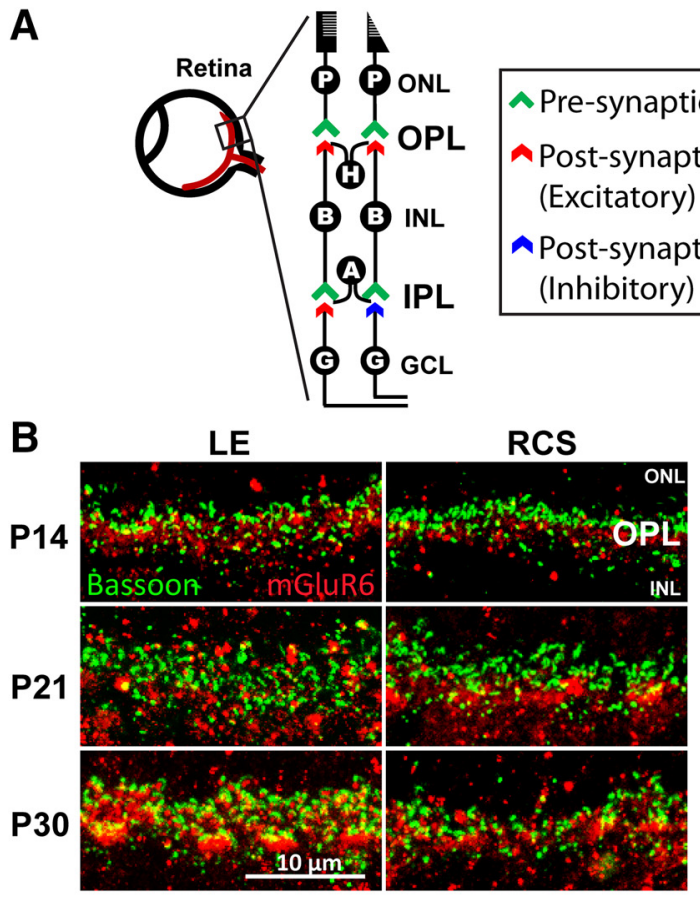

\section{D}
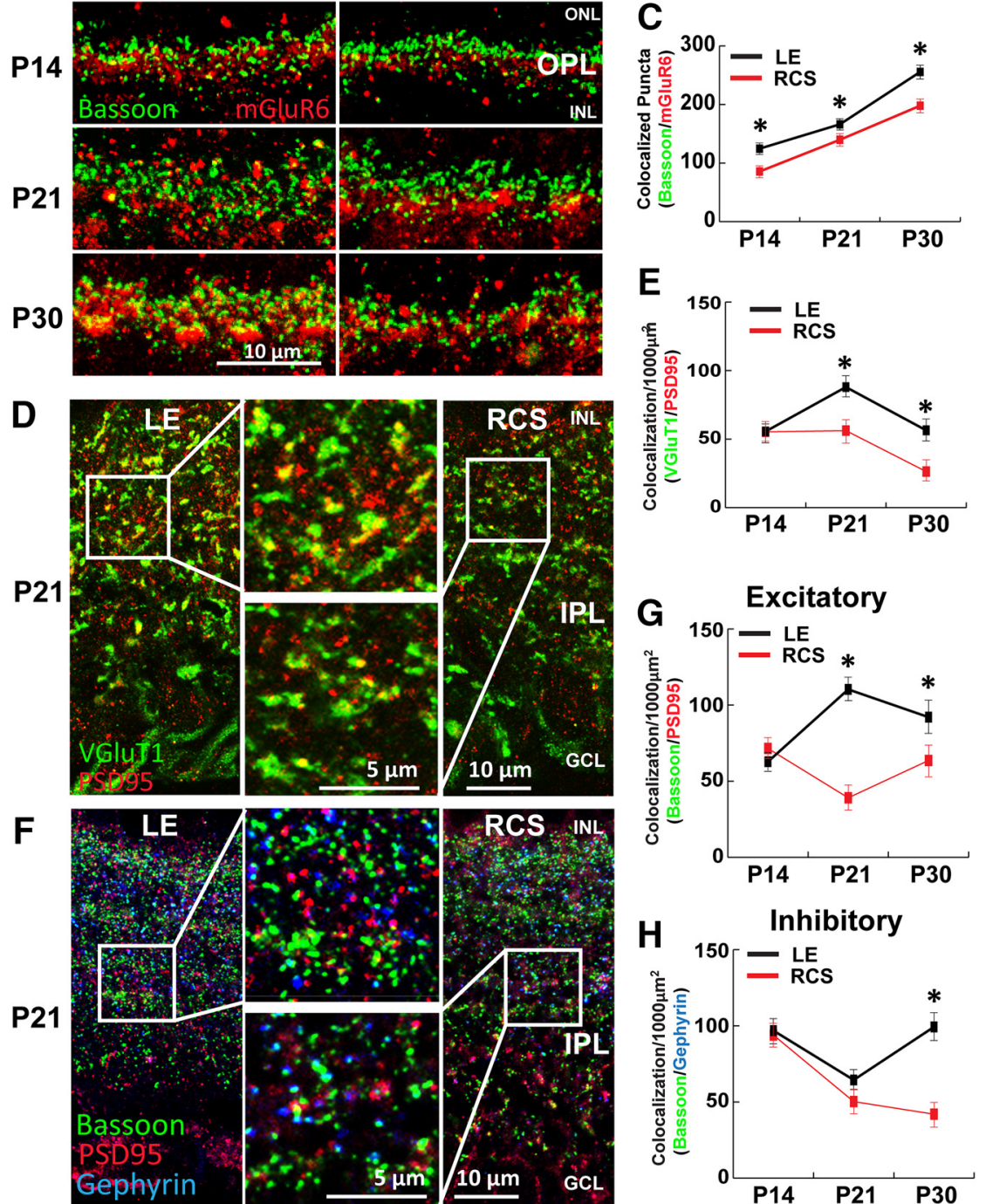

H
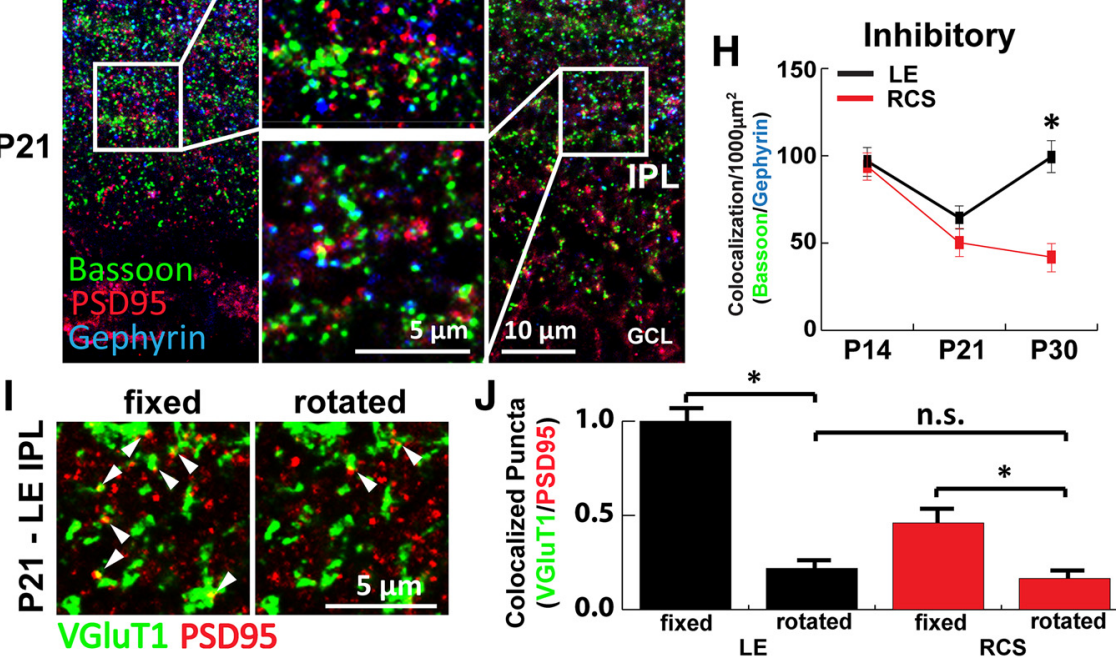$$
\text { P14 }
$$

n.s.

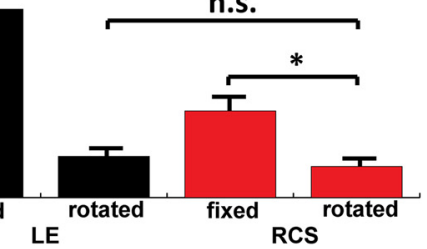

Figure 3. Synaptic development is impaired in RCS rats preceding PR loss. $A$, Schematic representation of the retinal layers. Presynapse (green) and postsynapse (red represents excitatory; blue represents inhibitory) are labeled in the synaptic layers. $B$, Representative images of the OPL with the PR ribbon synapses labeled for Bassoon (green) and mGluR6 (red) from LE (healthy) and RCS (degenerative) retinas on P14, P21, and P30. C, Quantification of ribbon synapses in the OPL between P14 and P30 revealed that synapse development in the RCS was already impaired on P14.D, Representative images of the IPL with the bipolar excitatory synapses labeled for VGluT1 (green) and PSD95 (red) from LE (healthy) and RCS (degenerative) retinas at P21. E, Quantification of the IPL of the RCS rats by staining the retinas with the presynaptic marker Bassoon, which marks both excitatory and inhibitory presynapses, in combination with antibodies specific for excitatory (PSD95) and inhibitory (Gephyrin) postsynapses (Fig. $3 F$ ). We quantified the number of excitatory and inhibitory synapses by counting the colocalization between Bassoon/PSD-95 or Bassoon/ Gephyrin, respectively. We found no significant differences in the numbers of either excitatory or inhibitory synapses between LE and RCS rats at P14 (Fig. 3G, $p=0.2981$; Fig. $3 H, p=0.6730$, Student's $t$ test). However, at $\mathrm{P} 21$, there is a sharp reduction in the numbers of excitatory synapses in the RCS rats, whereas the number of inhibitory synapses is comparable with LE controls (Fig. $3 G, p<$ 0.0001; Fig. $3 H, p=0.1840$, Student's $t$ test). By P30, we observed significantly fewer excitatory and inhibitory synapses in the RCS rat compared with LE controls (Fig. $3 G, p=0.0492$; Fig. $3 H, p<0.0001$, Student's $t$ test). To ensure that the colocalization we observe between presynaptic and postsynaptic markers are specific and are not due to chance, we reanalyzed the same images after we randomized the puncta by rotating one channel $90^{\circ}$ with respect to the other. In the randomized images, the colocalization was sharply diminished, confirming that the VGluT1/ PSD95 overlap we observe is not due to random chance (Fig. 3I, J; one-way ANOVA, $p<0.0001)$. These results show that formations of multiple classes of synapses are altered in the RCS rats. The excitatory synapse development is impaired before

\footnotetext{
bipolar excitatory synapses in the IPL between P14 and P30 revealed that synapse development in the RCS rat is compromised between P14 and P21. $\boldsymbol{F}$, Representative images of the IPL with the excitatory and inhibitory synapses labeled for Bassoon (pre, green), PSD95 (excitatory post, red), and Gephyrin (inhibitory post, blue) from LE (healthy) and RCS (degenerative) retinas at P21. Quantification of (G) excitatory and $(\boldsymbol{H})$ inhibitory synapses formed in the IPL between P14 and P30 revealed that deficits in excitatory synapse development occurred before deficits in inhibitory synapses. To demonstrate that the overlap in VGlut1 and PSD95 puncta is specific to synapses and is not due to random chance in densely stained tissue, confocal z stacks were split into two channels (VGlut1 and PSD95), the PSD95 channel was rotated $90^{\circ}$ out of alignment, then the two channels were remerged and analyzed for apparent colocalization of presynaptic puncta. $I$, Representative LE retina IHC image of fixed (original) and rotated image. J, For both LE and RCS rat retinas, overlap frequency was significantly decreased in the rotated images compared with the original images. Data were obtained from a minimum of 3 animals of either sex and expressed as mean \pm SEM. ${ }^{*} p<$ 0.05 .
} 
A P21 LE

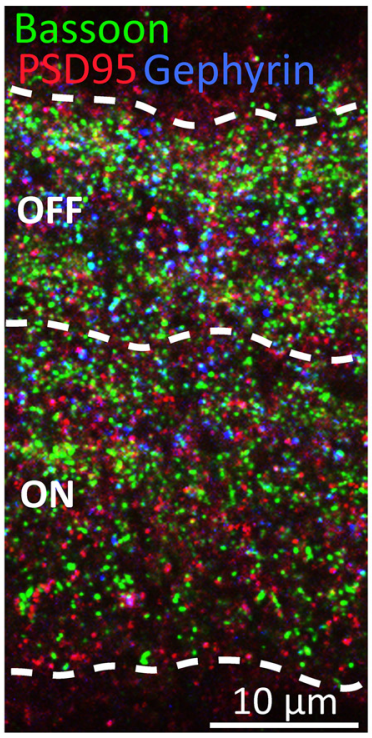

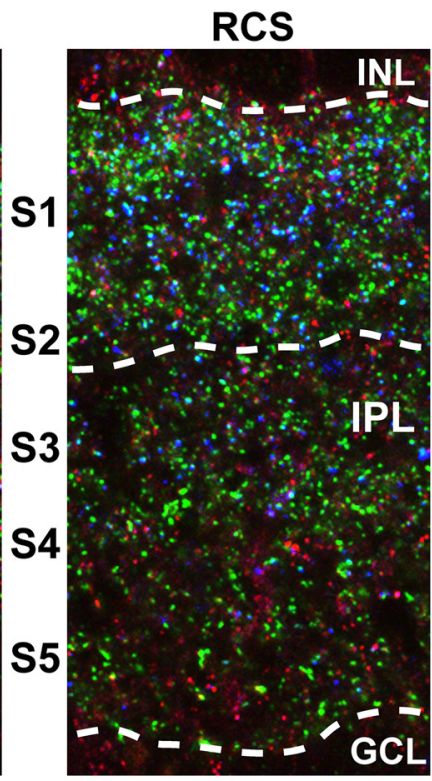

B

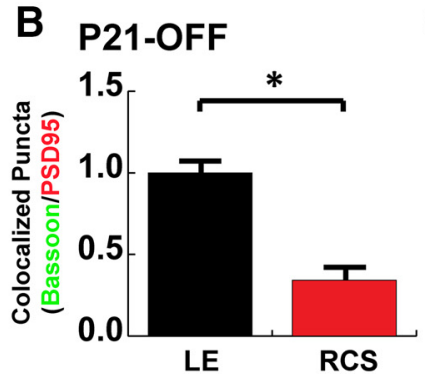

D P21-ON

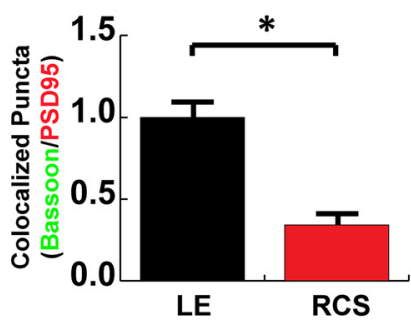

C P21-OFF

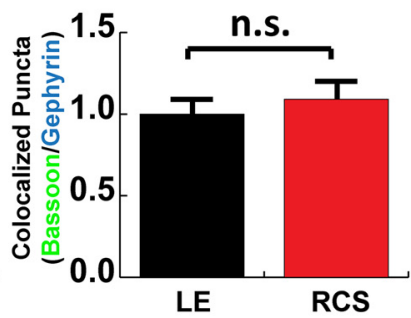

E P21-ON

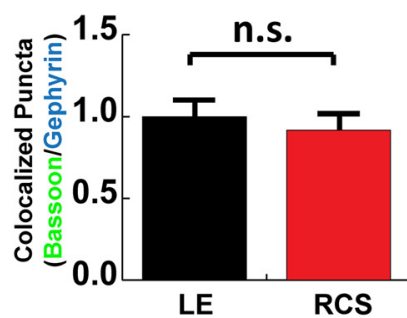

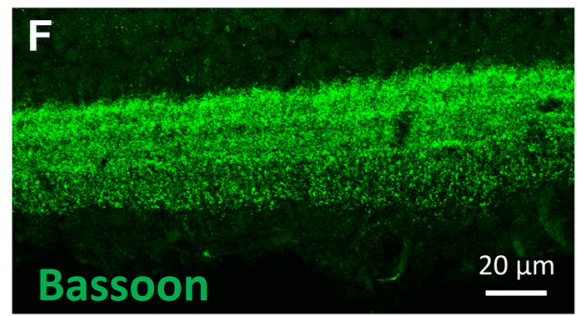
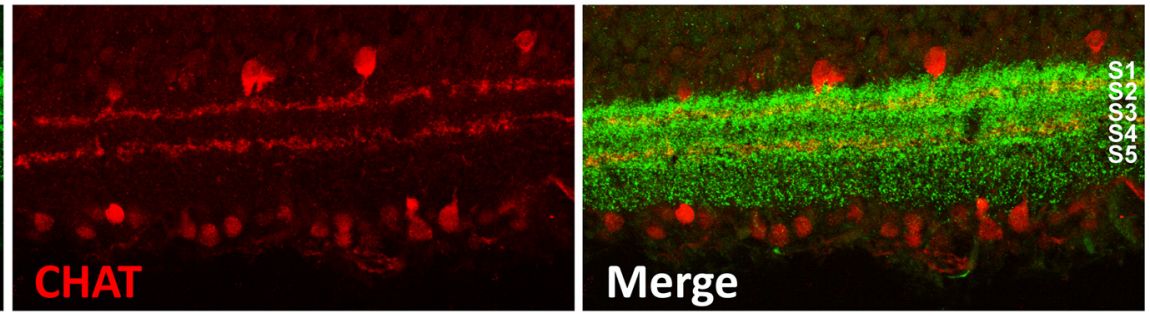

Figure 4. Synapses in both on- and off-sublaminae layers are developmentally impaired. $A$, Representative images in the IPL with the excitatory and inhibitory synapses labeled for Bassoon (pre, green), PSD95 (excitatory post, red), and Gephyrin (inhibitory post, blue) from LE (healthy) and RCS (degenerative) retinas on P21. OFF and 0N layers were identified by layered enrichment of Bassoon. Excitatory synapse quantification shows both $(\boldsymbol{B})$ OFF and $(\boldsymbol{D})$ ON layer have reduced number of synapses in RCS rats on P21, although there were no significant changes in the number of inhibitory synapses formed in both $(\boldsymbol{C})$ OFF and $(\boldsymbol{E})$ ON layers. $\boldsymbol{F}$, Representative P21 LE retina image of IPL costained with antibodies against Bassoon (green) and (hAT (red). Data were obtained from a minimum of 3 animals of either sex and expressed as mean \pm SEM. ${ }^{*} p<0.05$.

P21, whereas inhibitory synapses are affected by P30, only after significant PR death occurs.

The synaptic connections of the IPL can be approximately divided into two sublayers, namely, the ON and OFF layers (Fig. $4 A$ ), based on the response of these synaptic connections to light stimuli (Werblin and Dowling, 1969; Hoon et al., 2014). To determine whether the synaptic deficits we observed in P21 RCS rat retinas were specific to one of these sublayers, we quantified the numbers of synapses formed in ON and OFF layers at P21. These sublayers were identified by costaining one of the presynaptic markers, Bassoon, with ChAT (Fig. 4F). The excitatory or inhibitory synapses located in S1-S2 and S3-S5 were categorized as ON and OFF layer synapses, respectively. Our results demonstrated that excitatory synaptic development is impaired concurrently in both $\mathrm{ON}$ and OFF layers (Fig. $4 B, D$; Student's $t$ test, $p<0.0001$ ); however, there were no significant differences in the number of inhibitory synapses in either sublayer (Fig. $4 C, p=0.3374$; and Fig. $4 E, p=0.4045$, Student's $t$ test), paralleling the results obtained from the entire IPL (Fig. $3 H$ ).

Collectively, these results illustrate that excitatory synaptic development in RCS rat retinas is impaired by P21, before the onset of significant retinal degeneration. The deficits in excitatory synaptic development occur in both the OPL and IPL. Furthermore, these results suggest that signaling mechanisms that control retinal synapse development is impaired in the RCS rat retinas.

\section{MG exhibit altered morphology during early development} preceding PR loss

Astrocytes are powerful controllers of excitatory synapse formation in the brain by secreting multiple synaptogenic factors, including TSPs (Christopherson et al., 2005; Eroglu et al., 2009). However, in the retina, the MG undertake astrocytelike functions by extending their processes into the synaptic plexiform layers and positioning themselves at close proximity to the axons and dendrites. The MG processes span the retina and infiltrate the plexiform layers to clear synaptically released neurotransmitters and to maintain ion homeostasis. MG cellular processes can be labeled by staining with an antibody specific for GS (an important enzyme that is required for the ability of MG to convert glutamate to glutamine to be shuttled back to neurons), and the MG cell nuclei can be visualized by staining with an antibody against SOX9 (SRY-box 9; Fig. 5A).

Previous studies showed that, in the RCS retinas, MG progressively become "reactive" during the course of retinal degeneration (Härtig et al., 1995; Dyer and Cepko, 2000; Wang et al., 2005; Zhao et al., 2010; Fernández-Sánchez et al., 2015; Hippert et al., 2015). For example, analysis of retinal sections from RCS rats at P1 through P100 showed that MG become positive for GFAP as early as P35 (Kimura et al., 2000), an indication that reactive 

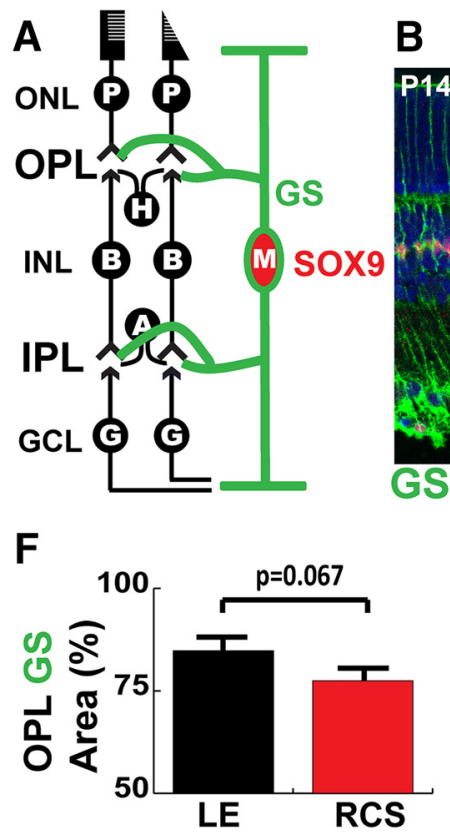
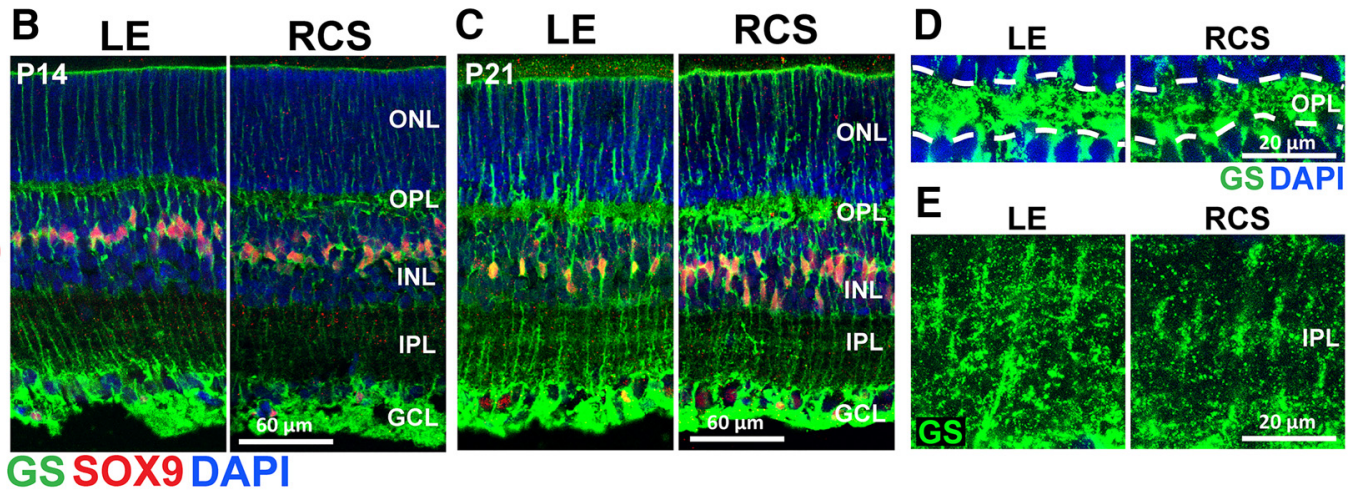
RCS
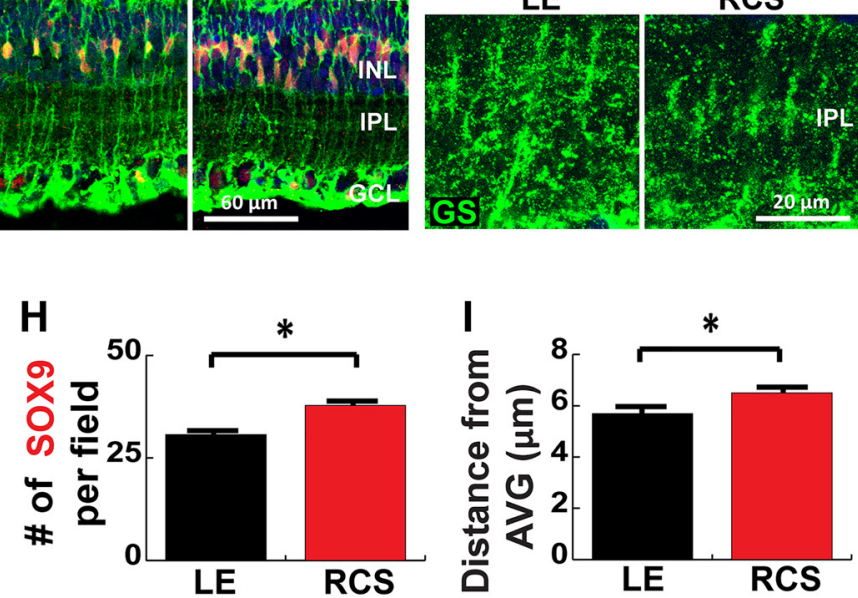

Figure 5. MG exhibit reactive morphology during early development preceding PR loss in the RCS rat. $A$, Schematic representation of the retinal layers and MG. Processes (GS, green) and nuclei (SOX9, red) of MG are labeled by IHC at (B) P14 and (C) P21. Representative pictures of GS ${ }^{+}$MG processes in (D) OPL and (E) IPL. The percentages of area covered by MG processes are reduced in both $(\boldsymbol{F})$ OPL and $(\boldsymbol{G})$ IPL in RCS rat. $\boldsymbol{H}$, Number of SOX9 ${ }^{+}$cell bodies and $(\boldsymbol{I})$ distance between SOX9 cell bodies were increased in RCS rats, suggesting proliferative changes of MG. Data were obtained from a minimum of 3 animals of either sex and expressed as mean \pm SEM. ${ }^{*} p<0.05$.

gliosis is initiated in RCS retinas at this age. On the other hand, no significant changes in the overall expression levels of GS were detected in RCS rats compared with healthy controls (Kimura et al., 2000). However, whether the number, distribution, and morphology of the MG are affected in RCS rats before the onset of GFAP expression is not known. To determine whether there are early changes in the MG of the RCS rats, preceding the onset of neuronal death, we immunostained age-matched LE and RCS retinas with antibodies against MG-specific markers, GS and SOX9, at P14 and P21. This analysis revealed that during the third week of postnatal development (P14-P21), the $\mathrm{GS}^{+} \mathrm{MG}$ processes become more arborized within the synaptic layers in LE rats; however, this arborization is impaired in the RCS rat (Fig. $5 B-E)$. We further analyzed the $\mathrm{GS}^{+} \mathrm{MG}$ process infiltration into the synaptic layers at P21 by quantifying the percentage area of the plexiform neuropil covered by $\mathrm{GS}^{+}$staining. We found that the percentage coverage of the neuropil by MG processes is significantly reduced in the IPL and trended toward a decrease in the OPL (Fig. 5F, $p<0.0001$; and Fig. 5G, $p=0.0351$, Student's $t$ test) of the RCS rats compared with LE controls. Furthermore, we found a significant increase in the number of MG (marked by SOX9) in the RCS rat retinas compared with LE WT rats (Fig. $5 H$; Student's $t$ test, $p=0.0351$ ), and the $\mathrm{SOX}^{+}{ }^{+}$nuclei appeared to have a disorganized distribution within the INL (Fig. $5 C$ ). In agreement with this, we found that the $\mathrm{SOX}{ }^{+}$cell nuclei were more broadly distributed within the INL, as determined by a slight, but statistically significant, increase in the average distance from the mean $\mathrm{SOX}^{+}{ }^{+}$nuclei position in the INL (Fig. 5I; Student's $t$ test, $p=0.0012$ ). Together, these findings indicate that MG in RCS rats display morphological and proliferative changes at P21, preceding significant PR loss. These changes in RCS MG coincide with the impaired synaptic development in these rats, suggesting that early activation of MG is linked to observed synaptic abnormalities.
Synaptogenic TSP1 and TSP2 are produced by MG, and their expression is reduced in the RCS rat retinas

We hypothesized that the synaptogenic signaling mediated by MG in the RCS rat retina is hampered, resulting in failure to promote synaptic development. Astrocyte-secreted TSP family proteins play an important role in excitatory synapse formation in the brain (Christopherson et al., 2005), and it has previously been reported that TSP-1 is also secreted by cultured MG in vitro (Yafai et al., 2014). Furthermore, IHC analyses of rat retina revealed the presence of TSP1 and TSP2 in the adult retina (Huang et al., 2013). TSPs are secreted proteins, and the signals for the proteins do not necessarily colocalize with the cell type that produces them. Therefore, to verify whether MG produce TSP1 and TSP2 in the rat retina, we used a combined approach of IHC and RNA FISH. We coimmunolabeled P21 LE retinal sections with an antibody against GS to mark MG and specific RNA FISH probes to localize the mRNAs that translate TSP1 and TSP2 (Thbs1 and Thbs2, respectively). The results demonstrated that, in the INL, where MG cell bodies are located, both Thbs1 and Thbs 2 mRNAs are localized to the cytoplasm of $\mathrm{GS}^{+}$cells (Fig. 6A). Interestingly, we also found Thbs1 and Thbs 2 to be highly enriched within the GS ${ }^{+}$ MG processes in the OPL (Fig. 6B). These results confirm that mRNAs for TSP 1 and TSP 2 are produced by MG in the rat retina. These results also suggest that Thbs 1 and Thbs 2 mRNAs, which are present in perisynaptic MG processes, are locally translated for direct secretion of TSPs into the OPL to control retinal synapse formation and maintenance.

To determine whether TSP expression is affected in the RCS retinas, we immunostained retinal sections from RCS and LE rats for TSP1 or TSP2 at P14 and P30, coinciding with early and late postnatal development (Fig. $6 C-J$ ). We found noticeable differences in both TSP1 and TSP2 expression between genotypes at both ages, although the reduction in TSP1 or TSP2 expression in RCS rats was most evident at P30 (Fig. 6G-J). Furthermore, we 

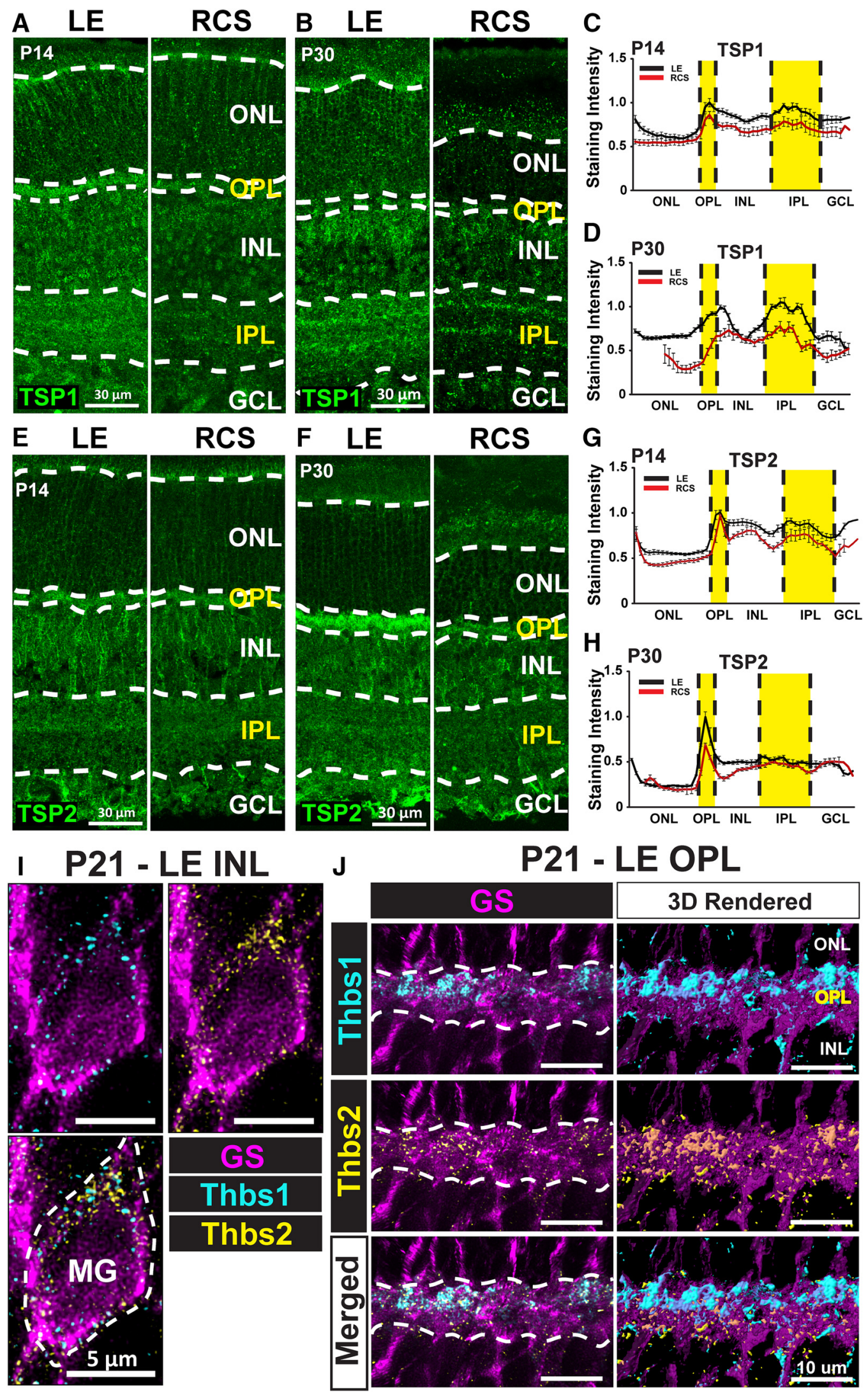

Figure 6. TSP1 and TSP2, expressed by MG, are reduced in RCS rat retinas. $A$, Representative images of the retina stained for TSP1 from LE (healthy) and RCS (degenerative) rats on P14 and (B) P30. Quantitative staining intensity analysis demonstrated that TSP1 is enriched in the synaptic layers, the expression was reduced in the RCS rat as early as (C) P14, and the expression gap became more distinct on $(\boldsymbol{D})$ P30. Representative images of the retina stained for TSP2 on $(\boldsymbol{E})$ P14 and $(\boldsymbol{F})$ P30. Quantitative staining intensity analysis demonstrated that TSP2 was enriched in the OPL, the expression was reduced in RCS rat as early as $(\boldsymbol{G})$ P14, and the expression gap became larger on $(\boldsymbol{H})$ P30. I, Confocal microscopy images showing fluorescent spots corresponding to Thbs 1 (cyan) and Thbs2 (yellow) mRNA in GS ${ }^{+}$cell bodies (dashed line) in rat retina. J, The Thbs 1 and Thbs 2 mRNAs were also enriched in the synaptic layers in GS ${ }^{+}$processes. $3 \mathrm{D}$ rendered images (right panels). 
A
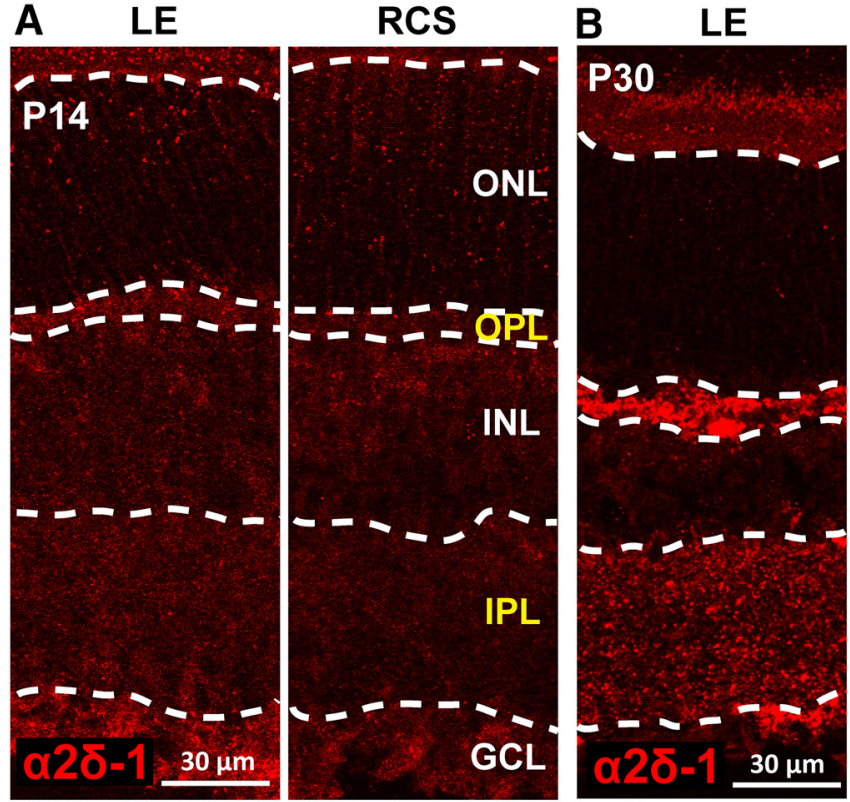

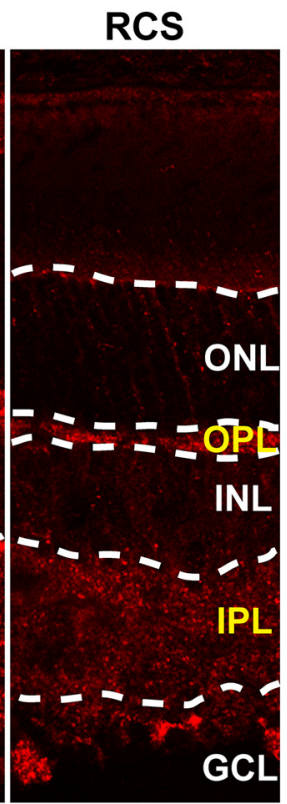

F LE IPL - P21
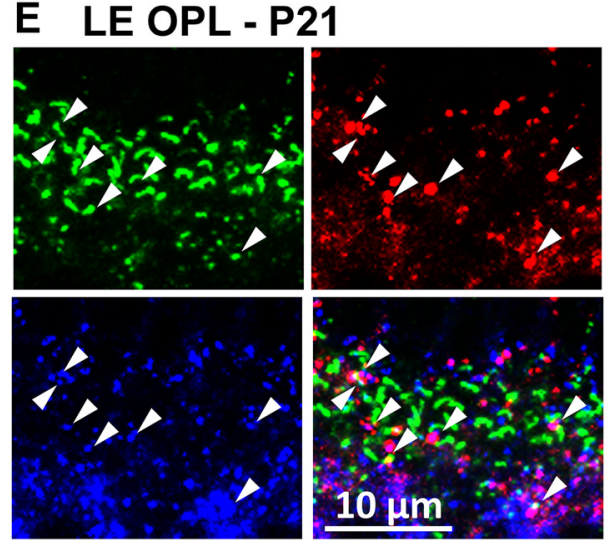

Bassoon $\alpha 2 \delta-1$ NR1

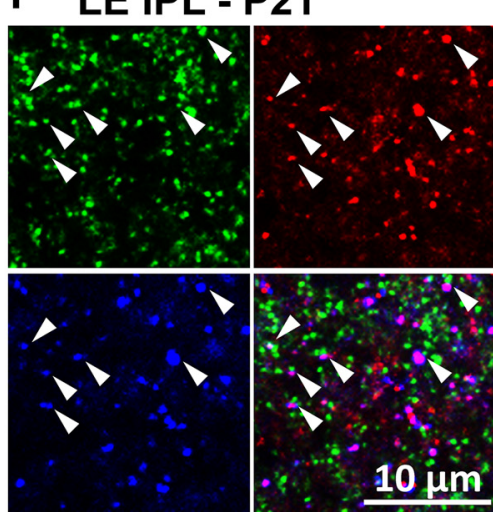

Bassoon a2ס-1 NR1
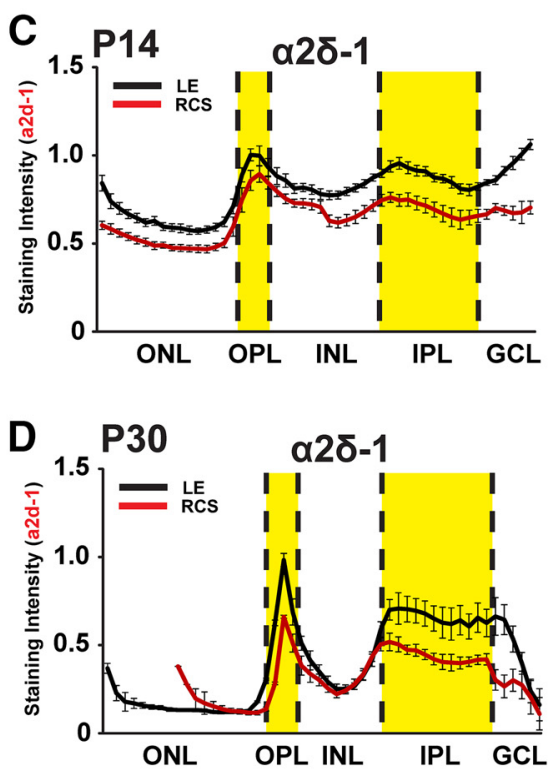

G LE IPL - P21

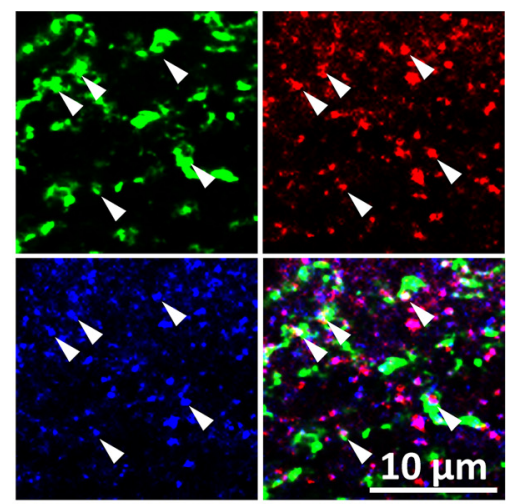

VGIUT1 a2ס-1 NR1

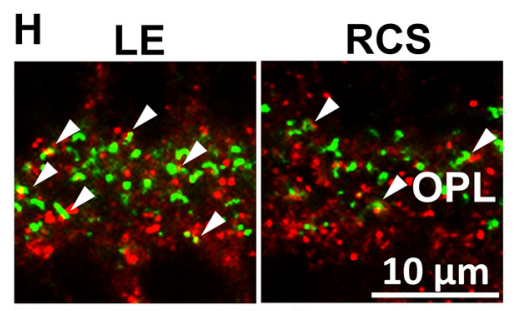

Bassoon $\alpha 2 \delta-1$

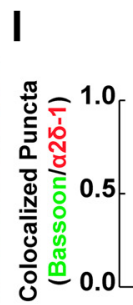

.

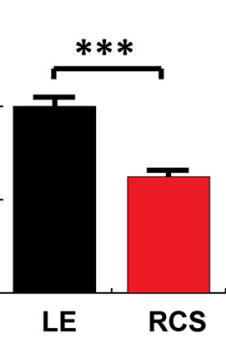

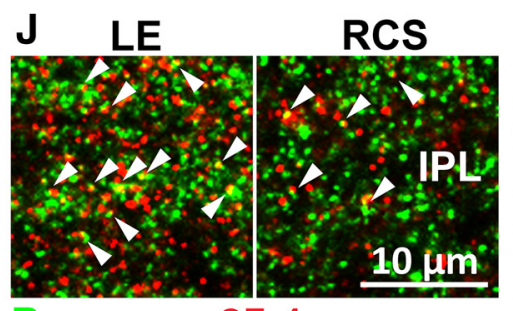

Bassoon $\alpha 2 \delta-1$

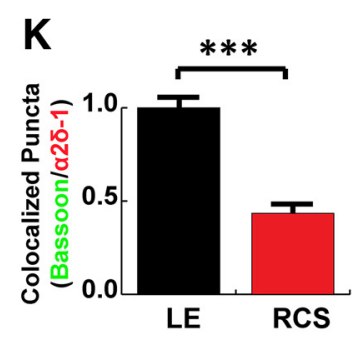

Figure 7. TSP-receptor $\alpha 2 \delta$ - 1 is synaptically expressed in the retina, and its expression is reduced in RCS rats. $A$, Representative images of the retina stained for $\alpha 2 \delta$ - 1 from LE (healthy) and RCS (degenerative) retinas at P14 and (B) P30.C, Quantitative staining intensity analysis demonstrated that $\alpha 2 \delta$ - 1 expression was reduced in RCS rat as early as P14. D, $\alpha 2 \delta$ - 1 was enriched in both the OPL and IPL, and the expression gap became more distinct at P30. Representative images of the $(\boldsymbol{E})$ OPL and (F and $\boldsymbol{G})$ IPL with the synapses labeled for Bassoon ( $\boldsymbol{F}$, green), VGluT1 ( $\mathbf{G}$, green), $\alpha 2 \delta$ - 1 (red), and NR1 (blue) from LE retina on P21 demonstrated postsynaptic expression of $\alpha 2 \delta$-1. Representative images of the $(\boldsymbol{H})$ OPL and ( $\boldsymbol{J}$ ) IPL synapses labeled for Bassoon (green) and $\alpha 2 \delta$ - 1 (red) from LE (healthy) and RCS (degenerative) retinas on P21. Quantification of $\alpha 2 \delta$-1-containing synapses formed in the (I) OPL and (K) IPL reveals that the number of $\alpha 2 \delta$ - 1 synapse is already reduced in RCS rat by P21. Data were obtained from a minimum of 3 animals of either sex and expressed as mean \pm SEM. ${ }^{* * *} p<0.0001$.

also detected differences in the localization of TSP1 and TSP2 in the retina. Even though we detected staining for both TSPs throughout all the retinal layers at P30, we found TSP1 to be enriched in the IPL (Fig. 6G,I), whereas TSP2 is enriched in the OPL (Fig. $6 H, J$ ). Together, these results show that TSP1 and TSP2 are expressed in rat retinas from early to late postnatal development coinciding with periods of extensive synapse development, and their expressions are reduced in RCS rat retinas.
TSP-receptor $\alpha 2 \delta$-1 is localized to retinal synapses, and $\alpha 2 \delta$-1-containing synapses are diminished in RCS rats

Our results strongly suggest that the synaptogenic signaling provided by MG-secreted TSPs is impaired in the RCS rat, resulting in deficits in synaptic development. TSPs interact with their synaptogenic receptor, calcium channel subunit $\alpha 2 \delta$-1, to promote excitatory synapse formation (Eroglu et al., 2009). Hence, the expression of $\alpha 2 \delta$-1 in the retina is necessary for TSP-mediated 
synaptogenesis. To determine developmental expression pattern of $\alpha 2 \delta$ - 1 in the rat retina, we used an antibody against $\alpha 2 \delta$-1 to examine the expression pattern in healthy LE rats. We found that the expression of $\alpha 2 \delta-1$ is sharply increased between P14 and P30 (Fig. 7A, B, left). In addition, we found $\alpha 2 \delta-1$ to be primarily localized to the OPL and IPL (Fig. $7 B$, left), where the TSPs are also enriched (Fig. 6). In contrast in the RCS retinas, $\alpha 2 \delta$ - 1 expression is not sharply increased between P14 and P30, resulting in diminished expression of $\alpha 2 \delta$-1 compared with age-matched LE controls (Fig. $7 A, B$, right). The staining intensity analysis between LE and RCS rats further confirmed enrichment of $\alpha 2 \delta$ - 1 in synaptic layers and decreased expression of $\alpha 2 \delta$ - 1 in the RCS rat retinas (Fig. 7C,D).

To determine whether $\alpha 2 \delta$ - 1 is localized to synapses, retinal sections from P21 LE rats were stained with an antibody against $\alpha 2 \delta-1$ in conjunction with pre(Bassoon, green) and postsynaptic (NMDA receptor subunit $1, \mathrm{NR} 1)$ markers. The results showed that $\alpha 2 \delta$ - 1 closely colocalizes with $\mathrm{NR} 1^{+}$postsynaptic terminals in both the OPL and IPL, whereas $\alpha 2 \delta$-1 puncta juxtaposes a subset of Bassoon positive presynaptic puncta (Fig. $7 E, F)$. These staining patterns suggest that $\alpha 2 \delta$ - 1 is predominantly localized to glutamatergic postsynapses in the retina. Importantly, quantitative analyses of $\alpha 2 \delta-1^{+}$synapses (marked as Bassoon $/ \alpha 2 \delta$ - 1 oppositions) in P21 LE and RCS rat retinas showed that $\alpha 2 \delta$-1-containing synapses are severely reduced in the IPL and OPL of RCS rats at this age before significant retinal degeneration occurs (Fig. $7 H-K$; Student's $t$ test, $p<0.0001$ ).

Together, our results show that TSP1 and TSP 2 and their neuronal receptor $\alpha 2 \delta$-1 are expressed in the synaptic layers of the rat retina during the time period of extensive excitatory synapse formation (i.e., P14-P30). This finding indicates a critical role for TSP signaling in the establishment of retinal synaptic connectivity during normal development. Our results also suggest that reduced TSP $/ \alpha 2 \delta$ - 1 signaling in RCS rat retinas may underlie impaired synapse formation.

\section{hUTC transplantation specifically preserves \\ $\alpha \mathbf{2} \delta$-1-containing synapses in RCS rats}

hUTC-secreted TSPs enhance synapse formation, maturation, and function in cultured retinal neurons in vitro (Koh et al., 2015). We found that in the neural retina MG secrete TSPs. Furthermore, we showed that TSP production and development of TSP receptor $\alpha 2 \delta-1^{+}$synapses are severely hampered in the RCS rats. Hence, we hypothesized that subretinal injection of hUTC may restore visual function by preserving synaptic connectivity and restoring $\mathrm{TSP} / \alpha 2 \delta$ - 1 signaling in the RCS retinas.

To test this possibility, we first quantified the number of OPL ribbon synapses in P95 LE rats (G1), RCS rats treated subretinally with BSS at P21(G4), RCS rats treated subretinally with hUTC at P21 (G3), or both at P21 and P60 (G6). To do so, retinal sections were stained with antibodies against the presynaptic marker Bassoon (green) and the postsynaptic marker mGluR6 (red) (Fig. $8 A$ ). As expected from visual function assessments (Fig. 1), P21
BSS-injected RCS rats (G4) displayed dramatic loss in their OPL ribbon synapses at P95 compared with the WT LE controls (G1, Fig. $8 A, C)$. Significantly higher numbers of OPL ribbon synapses were preserved in the RCS rat retinas that underwent hUTC administration at P21 (i.e., both G3 and G6). This result shows that, in addition to preserving PRs (Fig. 2E,F), hUTC injection at P21 also protects OPL ribbon synapse connectivity.

The preservation of ribbon synapses is not significantly different between animals receiving one (P21) or two (P21+ P60) injections (i.e., G3 and G6), respectively. However, the assessment of visual function revealed a marked improvement in the luminescence threshold responses of only the latter group (G6, Fig. 1E). To determine whether this difference could be explained by preservation of TSP $/ \alpha 2 \delta$ - 1 signaling in the OPL, we measured the number of synapses containing TSP receptor $\alpha 2 \delta$-1 (visualized by the colocalization of Bassoon and $\alpha 2 \delta-1$ ). We found that $\alpha 2 \delta$-1-containing synapses are specifically preserved only in the RCS rats that received P21 and P60 double injections (Fig. $8 B, D$; one-way ANOVA, $F_{(3,156)}=69.2185, p<0.0001$ ). This result strongly suggests that protection of $\alpha 2 \delta$-1-containing synapses may underlie enhanced functional recovery with the double injection treatment regimen.

To determine whether hUTC injections also affected synaptic connectivity in the IPL, we quantified the number of VGlut1/ $\mathrm{PSD}^{+}{ }^{+}$bipolar cell synapses of the same experimental groups: LE rats (G1), RCS rats treated subretinally with BSS at P21(G4), or RCS rats treated subretinally with hUTC at P21 (G3) or both at P21 and P60 (G6). Similar to the OPL, P21 BSS-injected RCS rats (G4) display a dramatic loss in their IPL bipolar cell synapses at P95 compared with the WT LE controls (Fig. 9A, $B$; one-way ANOVA, $\left.F_{(3,163)}=12.3739, p<0.0001\right)$. Subretinal injection of hUTC did not protect IPL bipolar cell synapses; however, double injection of hUTC (G6, P21 and P60) protected a large majority of the TSP-receptor $\alpha 2 \delta$-1-containing synapses in the IPL (Fig. 


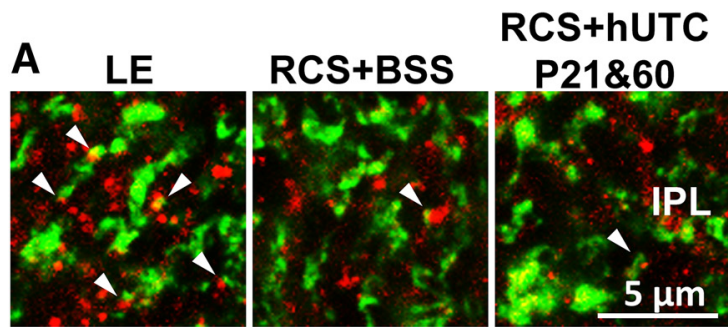

VGIUT1 PSD95
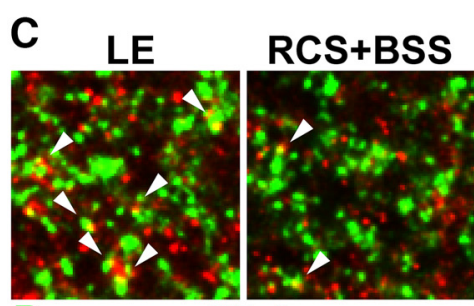

Bassoon $\alpha 2 \delta-1$

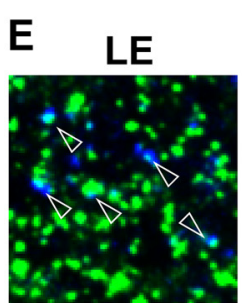

RCS+BSS

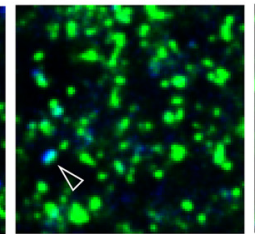

Bassoon Gephyrin
RCS+hUTC
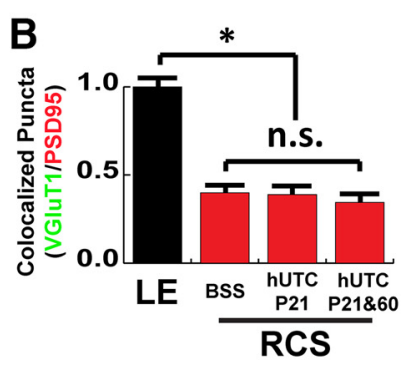

*
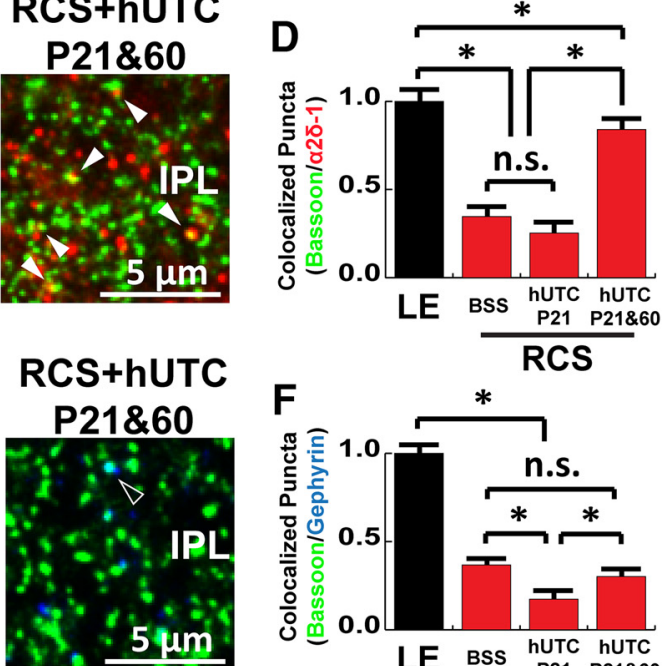

RCS+hUTC P21\&60

5 um
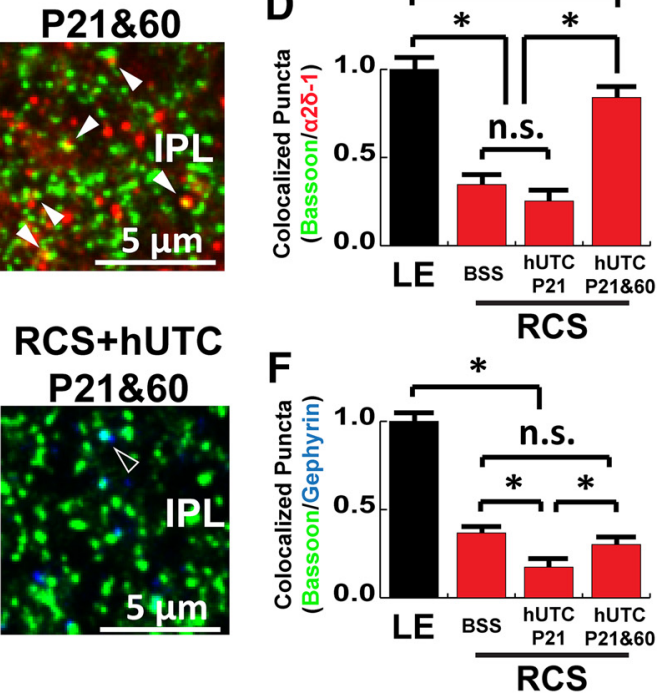

Figure 9. Subretinal hUTC transplantation preserves $\alpha 2 \delta$-1-containing synapses in the IPL of RCS rats. $\boldsymbol{A}$, Representative images of the IPL labeled for VGluT1 (green) and PSD95 (red) from LE (control), RCS + BSS, and RCS + hUTC P21 and P60 retinas on P95. $B$, Quantification of excitatory synapses in the IPL revealed that synapse numbers did not differ between RCS $+B S S$ and RCS + hUTC P21 and P60. C, Representative images of the IPL labeled for Bassoon (green) and $\alpha 2 \delta-1$ (red) from LE (control), $R C S+B S S$, and RCS + hUTCP21 and P60 retinas on P95.D, The $\alpha 2 \delta$-1-containing synapses were specifically preserved with hUTC transplantation. $\boldsymbol{E}$, Representative images of the IPL stained for Bassoon (green) and Gephyrin (blue) from LE (control), RCS + BSS, and RCS + hUTC P21 and P60 retinas on P95. $F$, hUTC transplantation did not preserve inhibitory synapses. Data were obtained from a minimum of 3 animals of either sex and expressed as mean \pm SEM. ${ }^{*} p<0.05$.

$9 C, D$; one-way ANOVA, $\left.F_{(3,167)}=82.9958, p<0.0001\right)$. However, subretinal injection of hUTC did not rescue inhibitory synapses visualized by the colocalization of Bassoon (presynaptic) and Gephyrin (postsynaptic; Fig. 9E, F).

Collectively, these results show that hUTC transplantation in RCS rats enhances synaptic connectivity. Importantly, we found that hUTC transplantation protects specific subsets of retinal synapses. In particular, repeated hUTC transplantation preserves synapses that contain TSP-receptor $\alpha 2 \delta$ - 1 in both OPL and IPL. These results suggest an important role for TSP $/ \alpha 2 \delta$-1- signaling in the proper connectivity and function of the retina and signify their importance as therapeutic molecular targets for treatment of retinal degeneration.

hUTC transplantation attenuates reactivity and preserves MG morphology

MG secrete TSPs and also provide important trophic and maintenance roles that enable proper retinal function (Williams et al., 2010). Therefore, we next tested whether hUTC injection improved MG morphology and function in the RCS rat retinas. To do so, we visualized MG in three groups of $\mathrm{P} 95$ rat retinas: that is, LE rats (G1), RCS rats treated subretinally with BSS at P21 (G4), and RCS rats treated subretinally with hUTC both at P21 and P60 (G6), by immunostaining with GS and SOX9 (Fig. 10A).

Interestingly, the RCS rats that received two injections of hUTC (G6) display significantly improved MG structure and GS expression compared with those treated with vehicle (BSS) (Fig. 10A). In the double injection group (G6), $\mathrm{GS}^{+} \mathrm{MG}$ processes localized to the outer limiting membrane (white arrow) maintained their tightly closed structure, which was comparable with healthy controls (LE). In contrast, the P21 vehicle-injected group (G4) showed abnormally extended and opened structures at the outer limiting membrane (Fig. 10A).

GS is a crucial enzyme that is required for the clearance and recycling of synaptically released glutamate. Loss of GS expression in astroglia is an indication of reactivity and loss of synaptic homeostasis (Härtig et al., 1995; Fernández-Sánchez et al., 2015; Hippert et al., 2015). We found that GS expression is preserved in the double hUTC-injected RCS rat retinas (G6), whereas vehicle-injected RCS rat retinas had a severe reduction in GS expression, particularly within the synaptic layers (Fig. $10 \mathrm{~B}, \mathrm{C}$; one-way ANOVA, $\left.F_{(2,78)}=34.7322, p<0.0001\right)$. The number of SOX9 ${ }^{+}$MG cell bodies was not different between the LE (G1) and vehicleinjected RCS rat groups (G4), but we observed a small but significant reduction in the number of SOX ${ }^{+}$cells in the double hUTC-injected group (G6, Fig. 10D; oneway ANOVA, $\left.F_{(2,24)}=3.6584, p=0.041\right)$. On the other hand, the double hUTC injection group (G6) displayed a lesser degree of disorganization of MG cell bodies in the INL as determined by a significantly reduced distance between MG cell bodies and the average $y$ axis of the INL (Fig. 10E; one-way ANOVA, $\left.F_{(2,445)}=15.1905, p<0.0001\right)$. This result further indicates that subretinal hUTC transplantation reduces reactive changes in the MG.

In agreement with this possibility, the reactive glia marker GFAP is absent from MG in P95 healthy control rat retinas (LE) but heavily upregulated in the vehicle-injected RCS rat group. Remarkably, double hUTC injection greatly subdued GFAP expression in the RCS rat retinas (Fig. 10E). These results show that repeated hUTC transplantation at $\mathrm{P} 21$ and $\mathrm{P} 60$ prevents reactive MG changes in RCS rat retinas, revealing a novel therapeutic effect for hUTC transplantation.

\section{Cell-autonomous loss of MERTK expression is sufficient to} induce reactive changes in MG

The RCS rat contains a deletion mutation in the gene that encodes MERTK. MERTK protein has well-established roles in regulation of phagocytosis and is expressed by the RPE cells (Feng et al., 2002); therefore, it is widely accepted that MERTK loss of function causes retinal degeneration by impairing the phagocytosis of the PR outer segment debris by RPE (D'Cruz et al., 2000; Nandrot et al., 2000). However, MERTK is also expressed by microglia and MG in the retina (Roesch et al., 2008, 2012; Siegert et al., 2012). Importantly, the expression level of MERTK in MG 


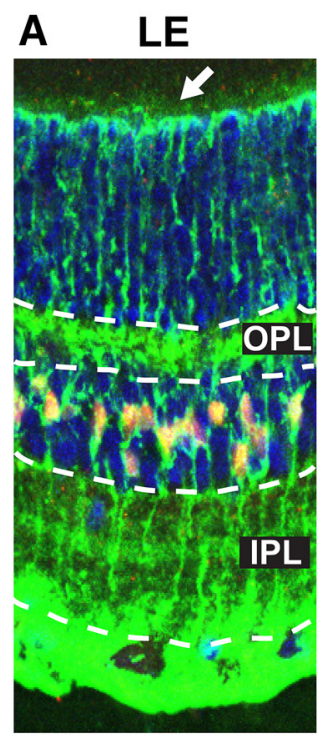

GS SOX9 DAPI

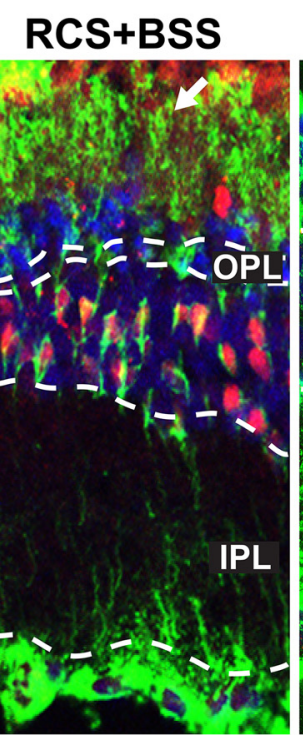

$\mathbf{F}$

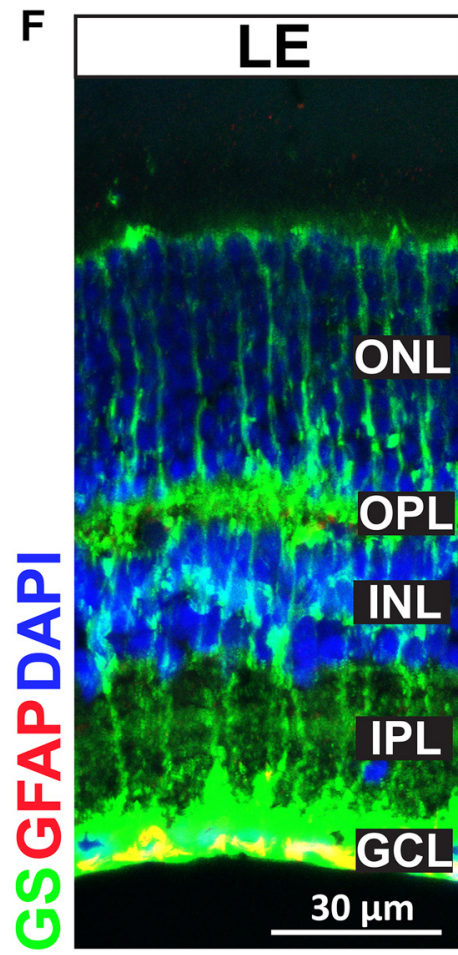

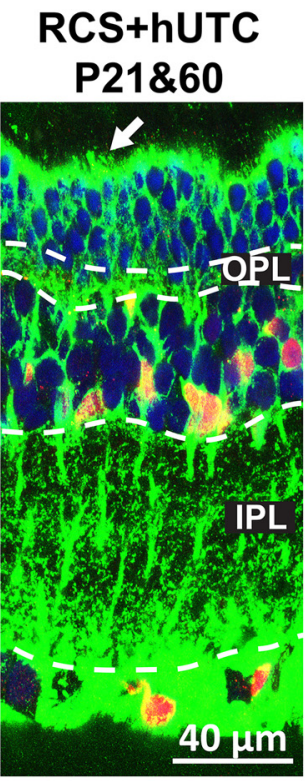
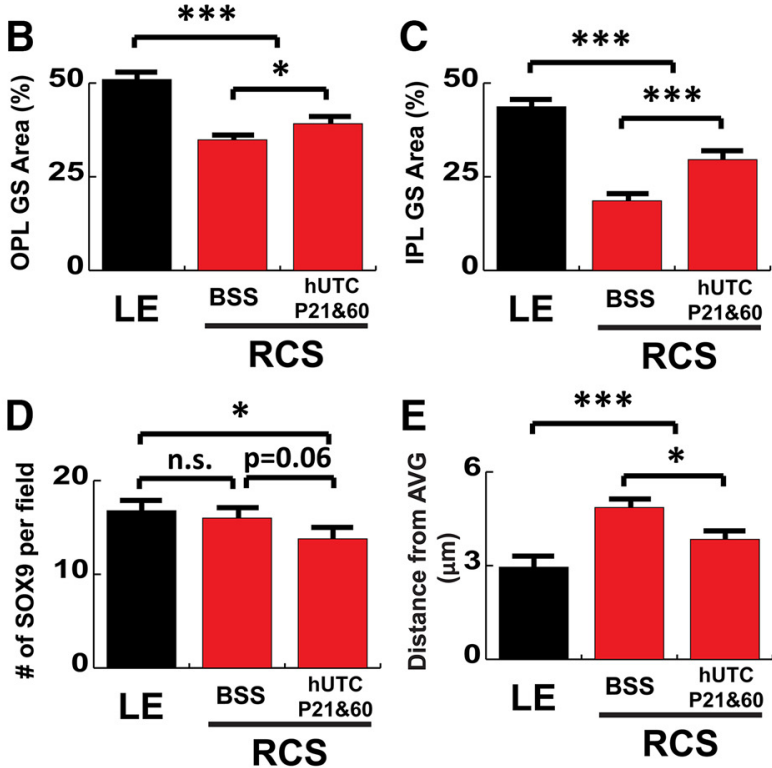

Figure 10. $\quad$ hUTC transplantation preserves MG morphology and attenuates reactivity. $A$, Representative images of the MG labeled for GS ( $g r e e n)$ and SOX9 (red) from LE (control), RCS + BSS, and $R C S+h U T C P 21$ and P60 retinae on P95. Percentages of $(\boldsymbol{B})$ OPL and $(\boldsymbol{C})$ IPL area covered by GS ${ }^{+}$processes were increased following hUTC transplantation in the RCS rat. The number of SOX9 ${ }^{+}$cell bodies $(\boldsymbol{D})$ distance between S0X9 cell bodies $(\boldsymbol{E})$ were decreased in RCS rat with hUTC transplantation, suggesting reduced proliferative change of MG. $\boldsymbol{F}$, Representative images of the MG labeled for GS (green) and GFAP (red) from LE (control), RCS + BSS, and RCS + hUTC P21 and P60 retinas on P95 demonstrate a sharply reduced reactive glial phenotype in the RCS + hUTC P21 and P60. Data were obtained from a minimum of 3 animals of either sex and expressed as mean \pm SEM. ${ }^{*} p<0.05,{ }^{* * *} p<0.0001$.

significantly increases with degenerative stress (Roesch et al., 2012). Thus, the loss of this gene in MG could have significant effects on the health and connectivity of the RCS rats. To test this possibility, we selectively ablated MERTK expression in MG of LE rats using CRISPR/SaCas9 system. To do so, we cloned an sgRNA against rat Mertk gene into a vector that contains SaCas9, a short Cas9 homolog that can be packaged into an AAV together with the sgRNA (Ran et al., 2015). AAVs were produced with a capsid protein that provides MG-selective transduction (Klimczak et al.,
2009). AAVs were intravitreally injected into the eyes of P7-P10 LE pups (Fig. 11 $A, B$ ). The left eyes were injected with the sgRNA targeting Mertk (MERTK KO), and the right eyes received the AAVs, which does not include an sgRNA, serving as controls (Fig. $11 C)$. We confirmed that the sgRNA specific for Mertk causes genetic mutations in the Mertk locus and reduces MERTK protein production in cultured astrocytes (Fig. $11 D, E$ ). Furthermore, IHC analyses demonstrated highly selective expression of HA-tagged SaCas9 in MG because the HA-tagged SaCas9 was 
A Muller glia selective AAV PaV-CRISPR A Muller glia selective AAV packaging vectors

$$
\begin{aligned}
& \text { REP }=\operatorname{CAP} \text { (Shh10) } \\
& \text { E4-E2a }-\mathrm{VA}
\end{aligned}
$$

\section{MERTK Targeting Vector}

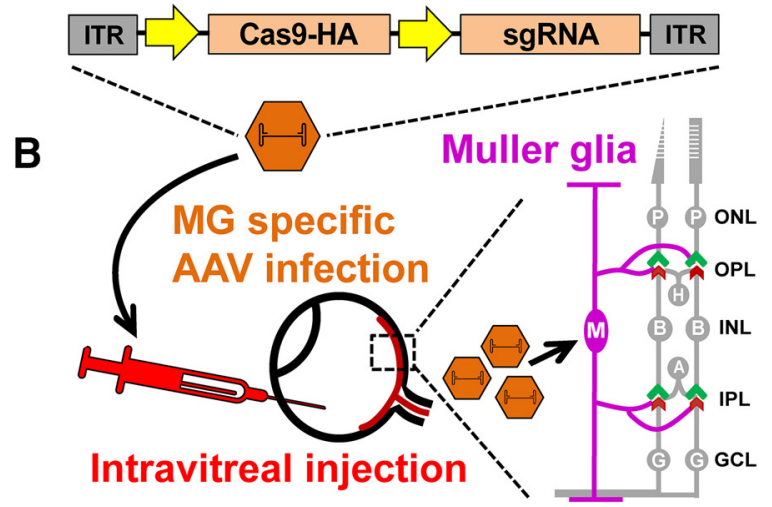

C

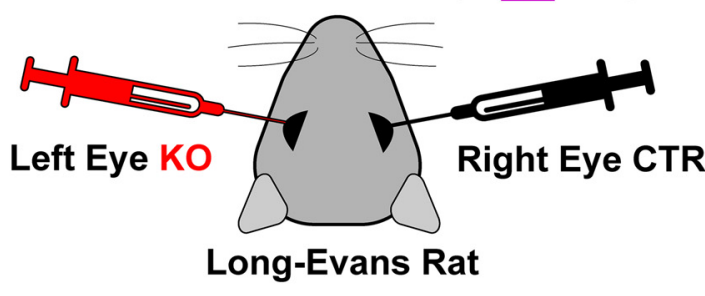

GS HA DAP

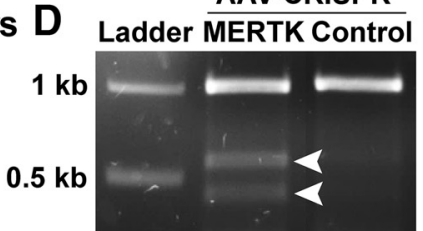

F Right Eye CRISPR-Control

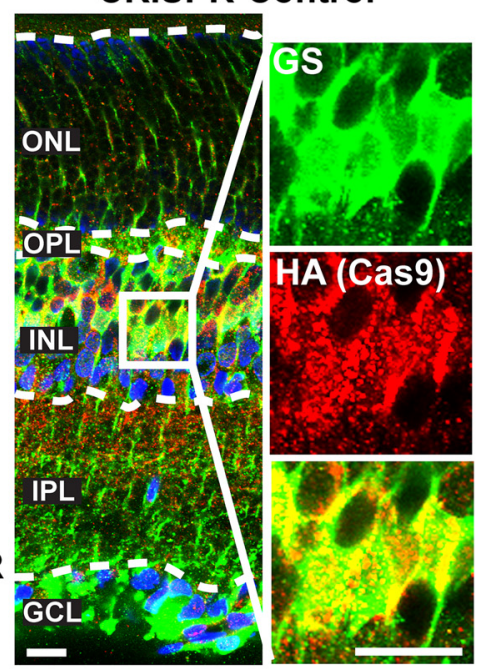

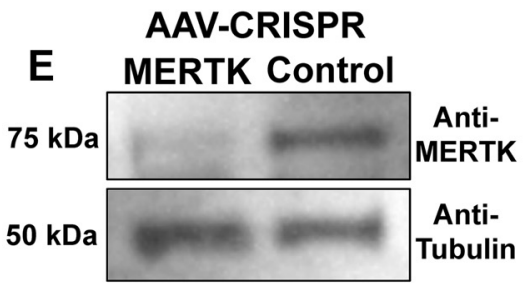

Left Eye CRISPR-MERTK

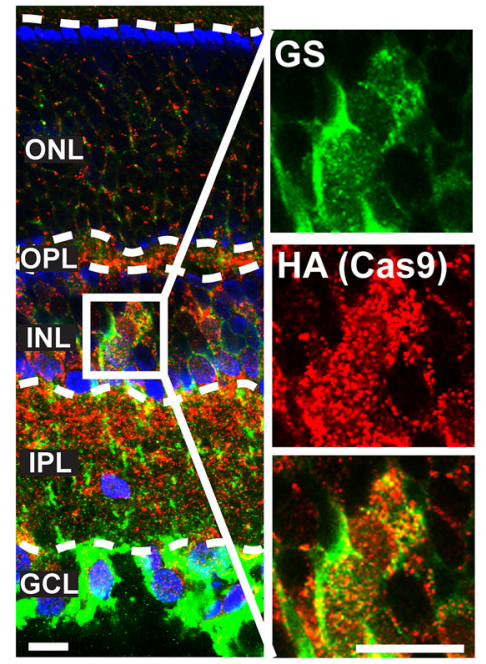

Figure 11. MG-specific deletion of MERTK using AAV-CRISPR/SaCas9 system. $\boldsymbol{A}$, To accomplish MG-specific MERTK deletion, CRISPR/SaCas9 vector targeting the MERTK gene is enveloped with shH10 mutant AAV capsid. B, AAVs are intravitreally delivered to target MG. C, In vivo intravitreal injection of AAV-CRISPR-MERTK (left eye) or AAV-CRISPR-Control (right eye). D, T7 Endonuclease I assay confirms CRISPR/SaCas9-induced cleavage in MERTK target sequence. Arrows indicate cleaved PCR amplicon. $\boldsymbol{E}$, Western blot of rat cortical astrocytes transduced with AAVs confirms reduced MERTK protein expression by CRISPR/SaCas9-AAV. F, Colocalization of GS (green, MG) and HA-tagged SaCas9 (red) demonstrates cell-type-specific transduction of CRISPR/ SaCas9-AAV. Scale bar, $10 \mu \mathrm{m}$.

exclusively enriched in the $\mathrm{GS}^{+} \mathrm{MG}$ cell bodies and processes in both left (treatment) and right eyes (control) (Fig. 11F).

Loss of MERTK specifically in MG did not cause any overt PR cell loss (Fig. 12). To determine whether knocking out MERTK in MG of LE rats induces reactive changes similar to what we observed in RCS rats, we quantitatively analyzed the expression of GS and the number and distribution of $\left(\mathrm{SOX9}^{+}\right) \mathrm{MG}$ cell bodies at P21 and P45 (Fig. 12A). We found a significant reduction in the infiltration of $\mathrm{GS}^{+}$processes in both synaptic layers in MGspecific MERTK KOs at P21, similar to our findings in the RCS rats (Fig. 5). At this age, there was already a significant reduction in the area covered by $\mathrm{GS}^{+}$staining in the IPL (Fig. 12C; Student's $t$ test, $p=0.0080$ ), whereas there was no significant change in the OPL (Fig. 12B; Student's $t$ test, $p=0.3402$ ) or the number of SOX9 between left and right retinas (Fig. 12D; Student's $t$ test, $p=0.6862)$. The reactive changes became more severe by $\mathrm{P} 45$, demonstrated by significantly reduced $\mathrm{GS}^{+}$area in both OPL (Fig. 12E; Student's $t$ test, $p<0.0001$ ) and IPL (Fig. 12F; Student's $t$ test, $p<0.0001$ ). These changes were coincident with increased number of SOX9 ${ }^{+} \mathrm{MG}$ cell bodies within the INL (Fig. $12 G$; Student's $t$ test, $p<0.0001$ ). The increased MG number was accompanied by atypical distribution of MG cell bodies. The disorganization was analyzed by comparing average SOX9 intensity profiles within the INL between left and right eyeball retinas (Fig. 12I). The results demonstrated that increased numbers of SOX9 ${ }^{+}$ MG at P45 had greater disorganization of MG cell bodies within the INL, whereas MG organization remained the same at P21 (Fig. 12J, K; ANCOVA, $p<0.0001)$. Together, these findings demonstrate that deleting MERTK in WT MG induces morphological and proliferative changes similar to the RCS rats, suggesting that MG dysfunction of the RCS rat may be independent of RPE dysfunction. It is important to note that, despite the severe reactive changes in MG upon MERTK deletion, the PR layer thickness did not appear to be affected in the MG-specific MERTK KO retinas.

Loss of MERTK in MG impairs retinal synapse development To determine whether loss of MERTK in MG is sufficient to cause synapse development problems similar to those observed in RCS rat retinas, we quantitatively analyzed the number of synapses in the inner and outer synaptic layers of right (control) and left (MERTK KO) retinas at P21 and P45. In the OPL, the number of ribbon synapses analyzed by the colocalization of Bassoon (presynaptic) and mGluR6 (postsynaptic) was significantly reduced in MERTK KO retinas compared with controls at both P21 and P45 (Fig. 13 A, B; Student's $t$ test, $p<0.0001$ ). We next analyzed IPL excitatory synapses based on colocalization of VGluT1 (presynaptic) and PSD95 (postsynaptic) and found that there was a significant defect during early development (P21); however, the defect was no longer detectible at P45 (Fig. 13C, $p<0.0001$; and Fig. $13 D, p=0.2323$, Student's $t$ test). Furthermore, $\alpha 2 \delta-1$ synapses determined by colocalization with Bassoon were severely reduced in both synaptic layers (OPL and IPL) and time points (P21 and P45; Fig. 13E-H). These results provide strong evidence that loss of MERTK in MG could significantly contribute to diminished visual function in retinal degeneration caused 
A

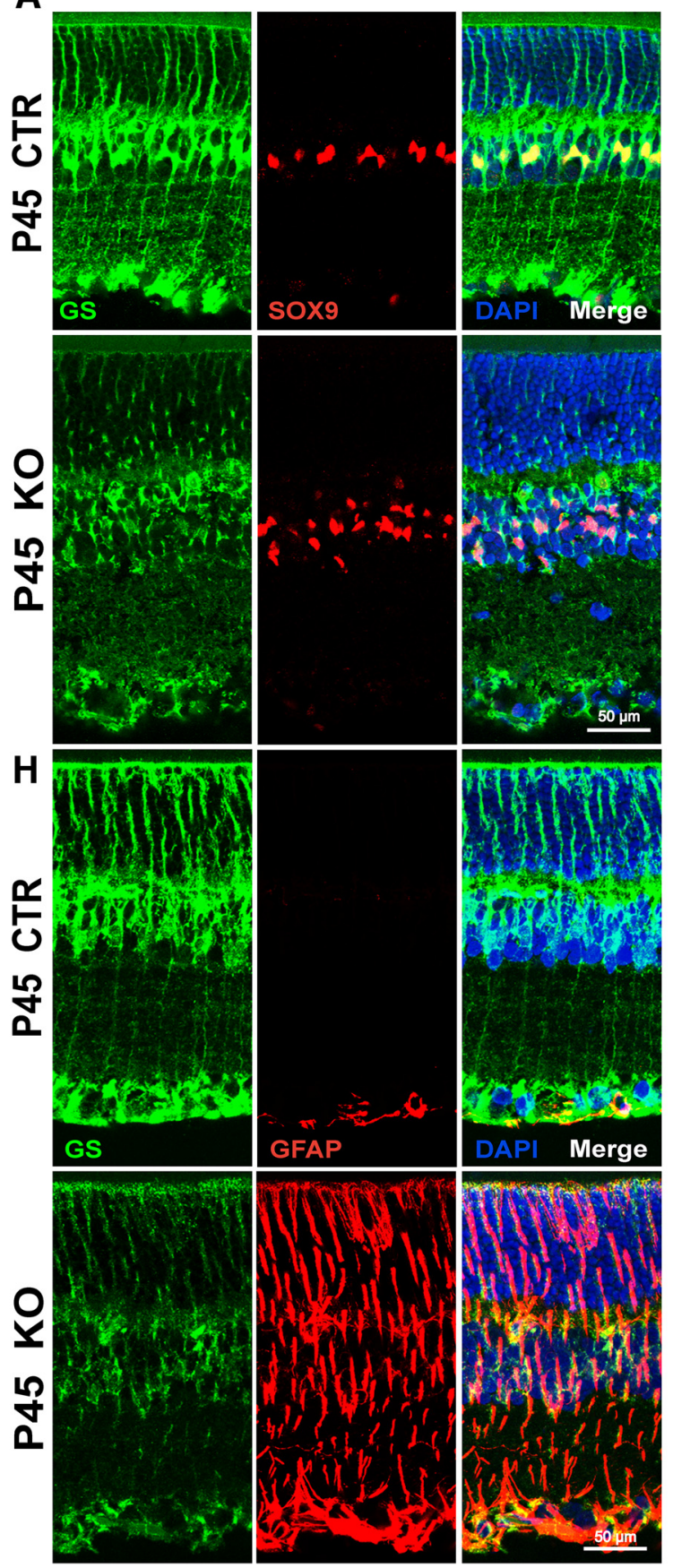

P21

B

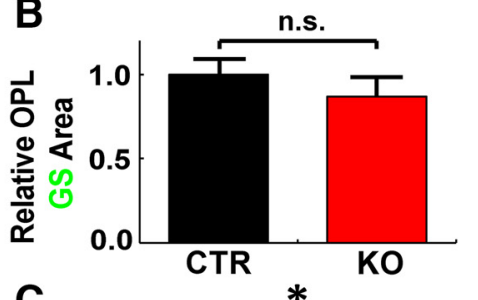

C

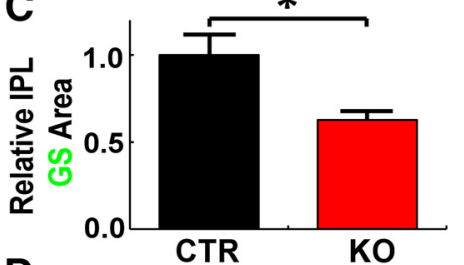

D

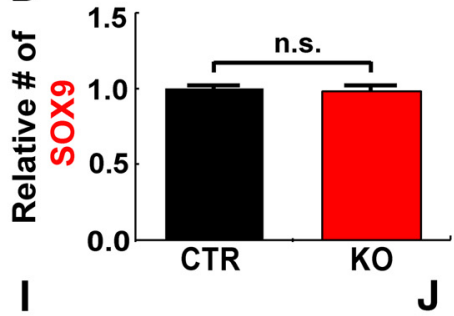

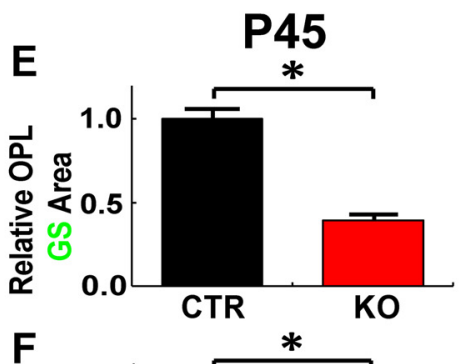

$F$
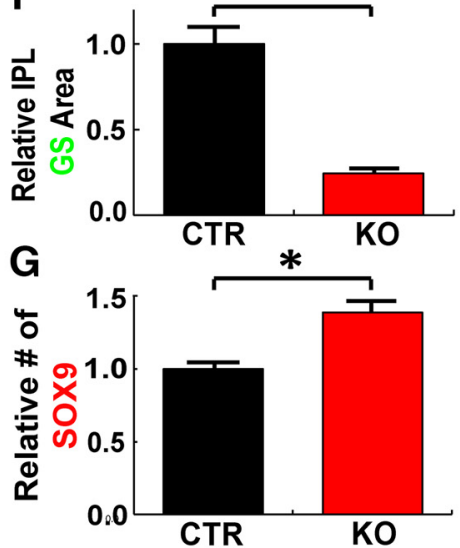
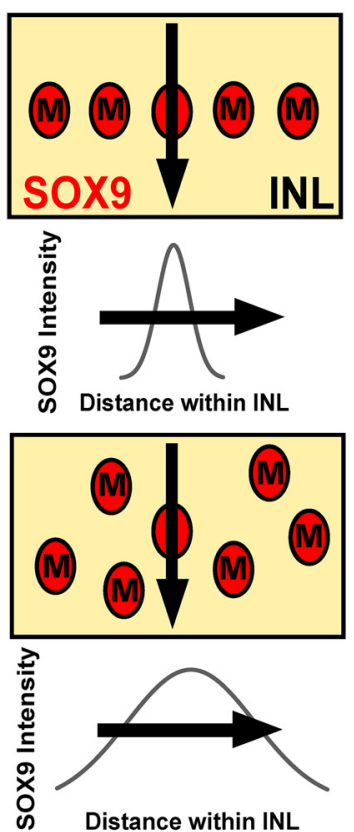

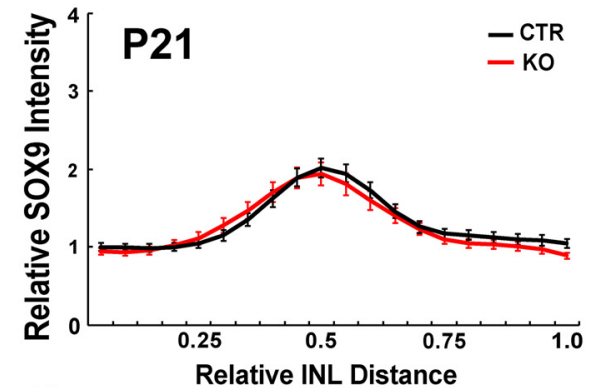

$\mathrm{K}$

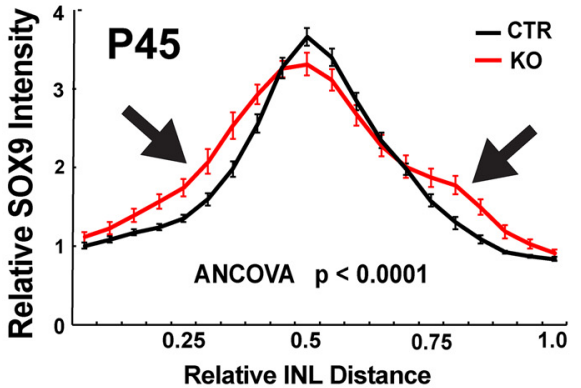

Figure 12. Loss of MERTK in MG induces reactive changes. A, Representative images of MG labeled with GS (green) and SOX9 (red) from P45 CTR (injected with Control-AAV, which only contains $\mathrm{SaCas9}$ ) or P45 $\mathrm{KO}$ (injected with AAV containing (RISPR against Mertk). Loss of $\mathrm{SS}^{+} \mathrm{MG}$ processes and disorganized SOX9 ${ }^{+} \mathrm{MG}$ nuclei in the INL are easily noticeable at this time point. Percentages of OPL and IPL area covered by GS ${ }^{+}$MG processes in MERTK-KO retinas compared with CTR $(\boldsymbol{B}, \boldsymbol{C})$ at P21 and $(\boldsymbol{E}, \boldsymbol{F})$ at P45 $(\boldsymbol{D}, \boldsymbol{G})$. $(\boldsymbol{H})$ GFAP (red) expression is sharply increased in P45 MERTK-K0 retinas. The number of $\mathrm{SOX9^{+ }}$ cell bodies is increased. $\boldsymbol{I}-\boldsymbol{K}$, The distribution of $\mathrm{SOX} 9$ cell bodies is disorganized in P45 MERTK-KO retinas, suggesting proliferative and reactive changes in MG. The disorganization was analyzed by measuring average SOX9 intensity profiles within the INL, then comparing between CTR and KO retinas. All data were obtained from a minimum of 3 animals of either sex and expressed as mean \pm SEM. ${ }^{*} p<0.05$.

by MERTK mutations by impairing the formation and/or maintenance of retinal synapses.

MERTK KO impairs synaptogenic function of cultured retinal glia

Astrocyte-secreted synaptogenic proteins, such as TSP1 and TSP2, induce formation of excitatory synapses between purified RGC cultures (Christopherson et al., 2005). We next used this cell culture system to test whether loss-of-function mutations in MERTK impair synaptogenic activity of retinal glia. To do so, we first isolated rat retinal glia (Trachtenberg and Packey, 1983), which then were treated with the same control or MERTK-targeting CRISPR/SaCas9 AAVs (as described in Fig. 11) for 5 d. MERTK KO or control retinal glia were next cocultured with RGCs for another $6 \mathrm{~d}$. The number of synapses formed between RGCs was assessed by the colocalization of Bassoon (presynaptic) and 

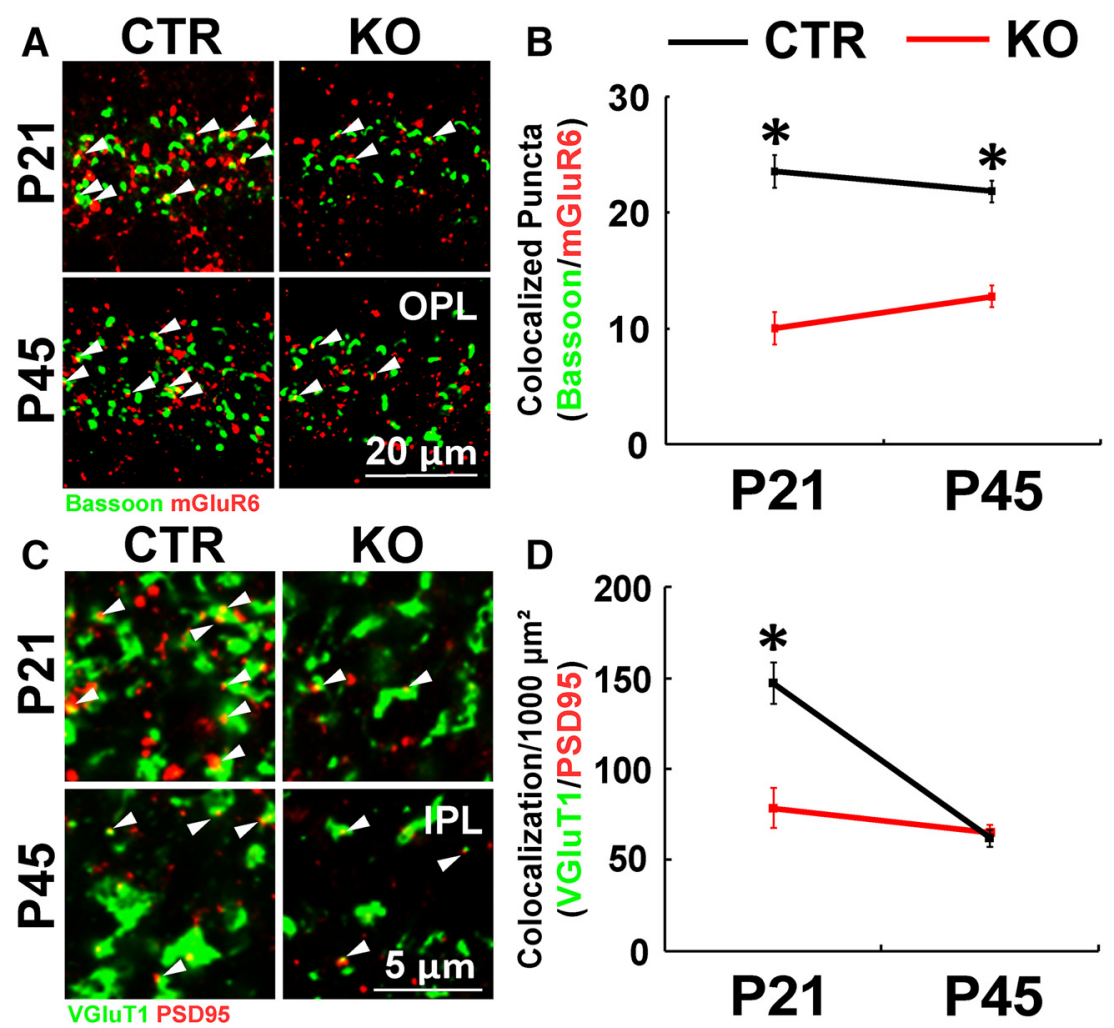

\section{D}
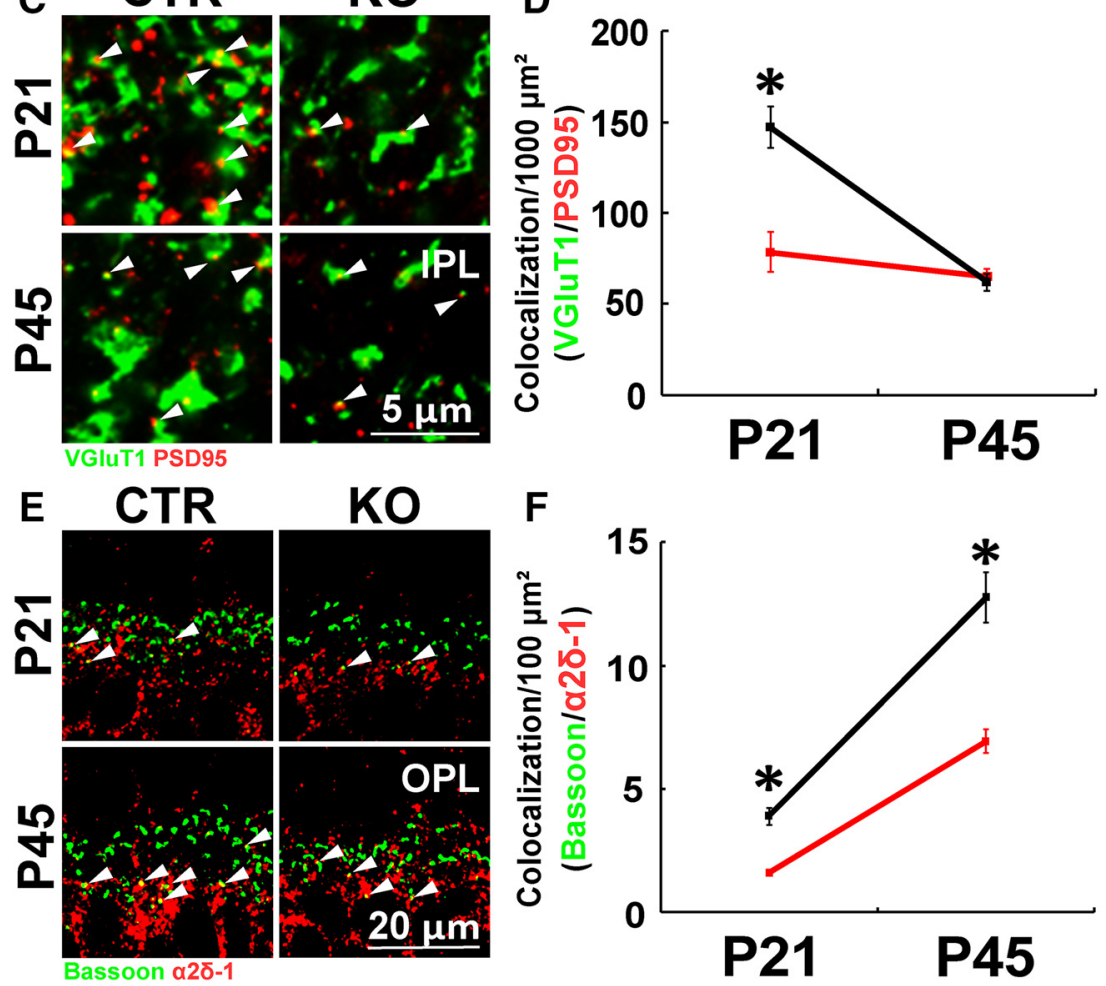

F
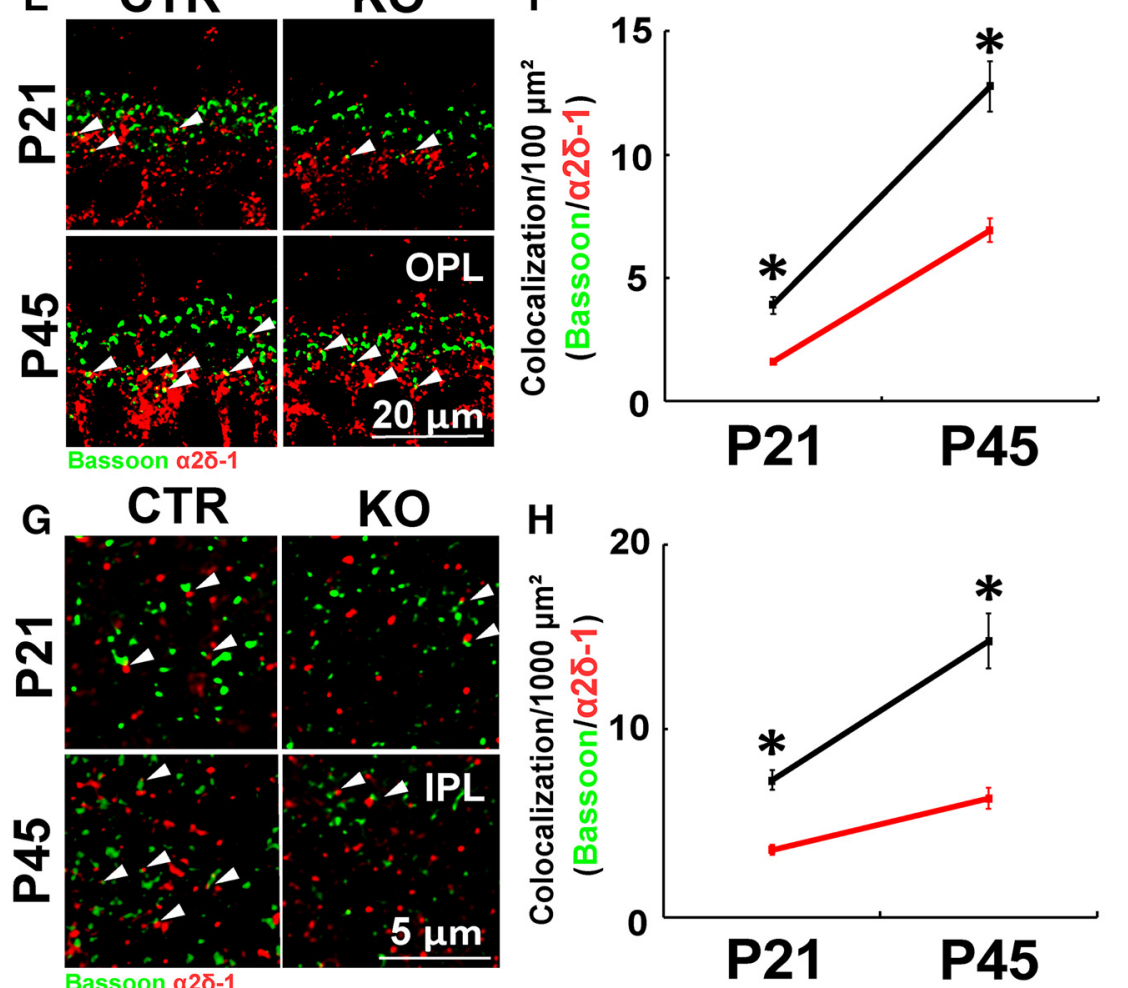

Figure 13. $\mathrm{KO}$ of MERTK in MG results in impaired synapse development in vivo. $\boldsymbol{A}$, Representative images of the OPL synapses labeled by Bassoon (green) and mGluR6 (red) from Control (CTR, right eyeballs) and MERTK K0 (K0, left eyeballs) at P21 and P45. $\boldsymbol{B}$, Quantification of synapses in the OPL revealed that synapse development is impaired in MG-specific MERTK-KO at both P21 and P45. C, IPL excitatory synapses are visualized by VGluT1 (green) and PSD95 (red). D, Quantification of synapse number demonstrates that early synaptic development at P21 is significantly affected by MERTK K0 but not at P45. Representative images of the (E) OPL and (G) IPL synapses labeled for Bassoon (green) and $\alpha 2 \delta-1$ (red). Quantification of the number of $\alpha 2 \delta$-1-containing synapses in both $(\boldsymbol{F})$ OPL and $(\boldsymbol{H})$ IPL revealed that KO of MERTK in MG induces severely reduced number of $\alpha 2 \delta$-1-containing synapses at P21 and P45. Data were obtained from a minimum of 3 animals of either sex and expressed as mean \pm SEM. ${ }^{*} p<0.05$.

Homer (postsynaptic) as previously described (Koh et al., 2015) (see also Materials and Methods). Retinal glia strongly induced excitatory synapse formation between RGCs compared with RGCs cultured alone (Fig. 14D; one-way ANOVA, $\left.F_{(2,87)}=14.2296, p<0.0001\right)$. However, the MERTK-KO retinal glia did not induce significant excitatory synapse formation (Fig. 14D). To confirm that synaptogenic effect of retinal glia was mediated via secreted factors, the CRISPR/SaCas9 AAVtreated retinal glia were cultured separately and conditioned media (CM) were collected as previously described (Christopherson et al., 2005; Koh et al., 2015). Then RGCs were treated with control or MERTK KO retinal glia-conditioned medium for $6 \mathrm{~d}$ (Fig. 14B). Because astrocytes are known to secrete multiple synaptogenic factors (Ullian et al., 2001), astrocyte CM was used as a positive control. The results demonstrated that addition of retinal glia CM is sufficient to induce excitatory synapse formation, comparable with astrocyte $\mathrm{CM}$ treatment (Fig. 14E; one-way ANOVA, $F_{(3,116)}=$ 9.3117, $p<0.0001)$. Importantly, retinal glia CM collected from MERTK KO cells did not promote synaptogenesis, which further indicates that deletion of MERTK from the retina glia impairs synaptogenic activity of these cells (Fig. 14E).

hUTC-secreted factors recover synaptogenic function of MERTK KO retina glia

Because subretinal hUTC transplantation attenuates reactivity of $\mathrm{MG}$ and rescues retinal synaptic connectivity in vivo (Figs. $8-10$ ), we tested whether hUTC secrete factors that could rescue synaptogenic function of the MERTK KO retina glia. In our previous study, we found that hUTC secrete synaptogenic TSP1, TSP2, and TSP4 (Koh et al., 2015), which may directly influence synaptic rewiring in vivo, or may signal to MG to regulate their synaptogenic functions. Therefore, we also examined whether UCM from hUTC in which TSP1, TSP2, and TSP4 were knocked down could still rescue the impaired synaptogenic function of the MERTK KO retina glia.

To test these possibilities, MERTK KO retina glia were first treated with UCM for 5 d before coculture with RGCs. UCM were collected from hUTC transduced with lentiviruses containing either scrambled shRNAs or a combination of TSP1, TSP2, and TSP4 targeting shRNAs as previously described (Koh et al., 2015). The transwell inserts in which the retinal glia were treated with UCM were thoroughly 
A Co-culture

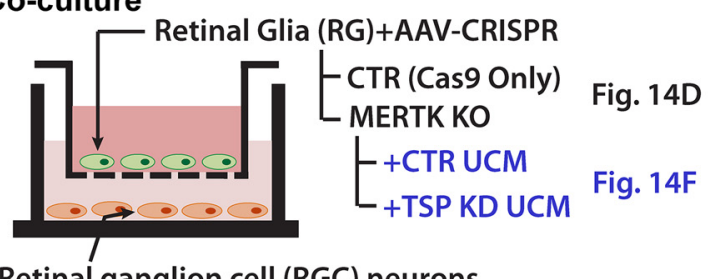

Retinal ganglion cell (RGC) neurons
B

Conditioned Medium (CM)

* AAV-CRISPR RG Conditioned Medium (RGCM)

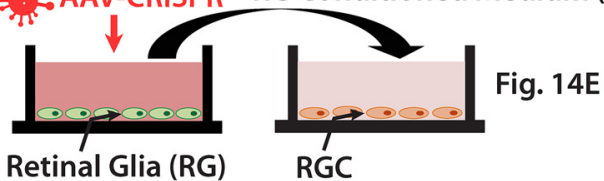

C
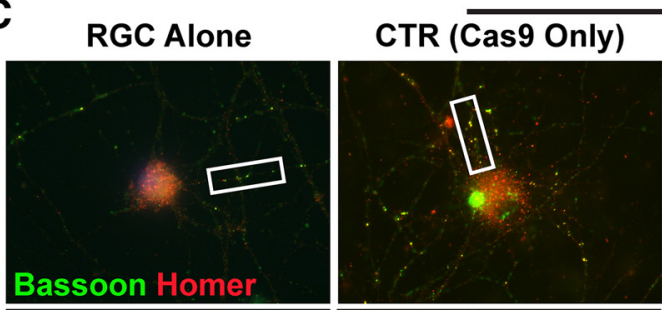

Co-cultured with Retinal Glia
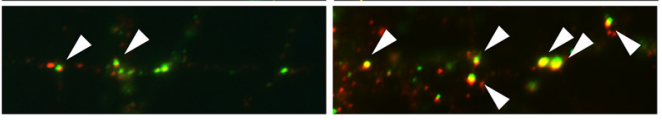

$10 \mu \mathrm{m}$

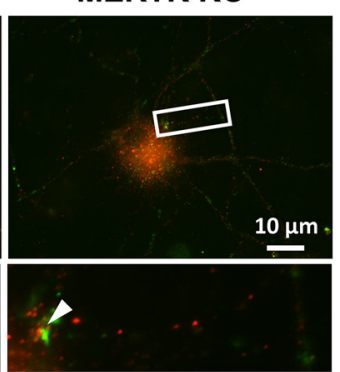

MERTK KO + UCM MERTK KO + KD UCM

D

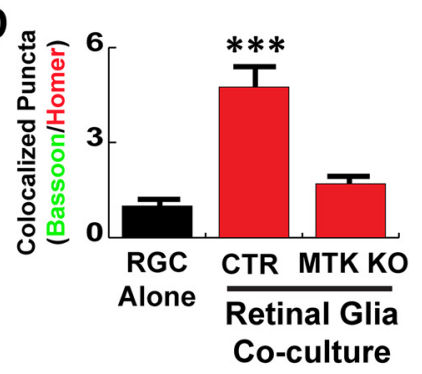

E

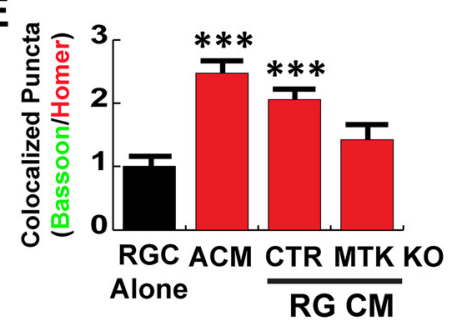

$\mathbf{F}$
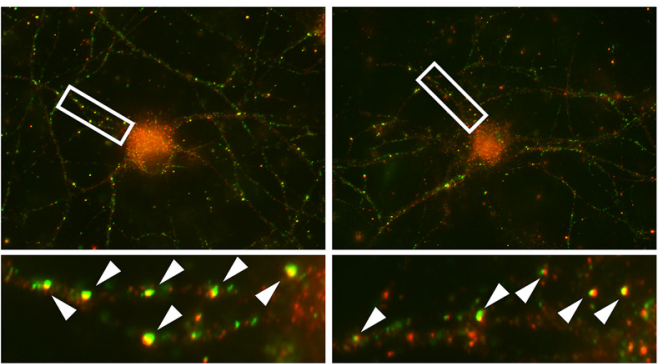

$p=0.17$
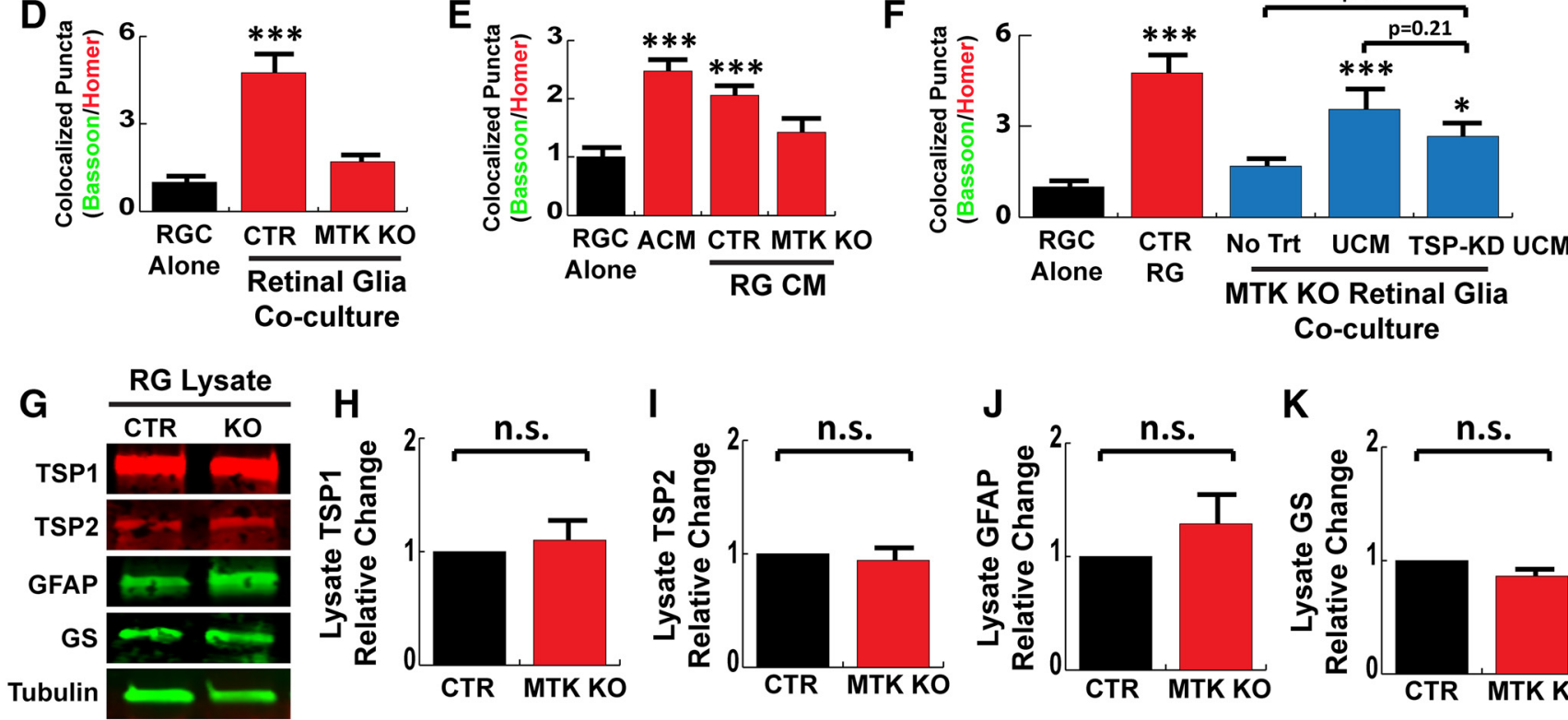

I
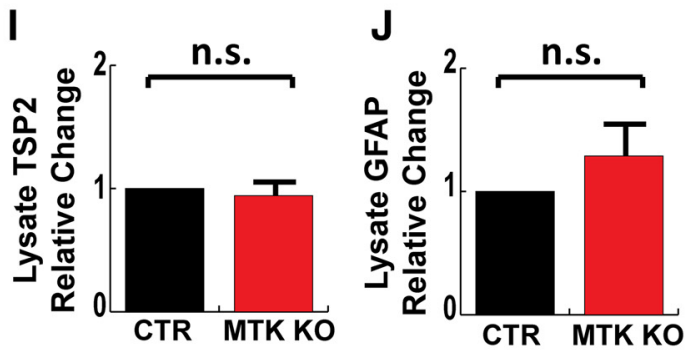

$\mathbf{K}$
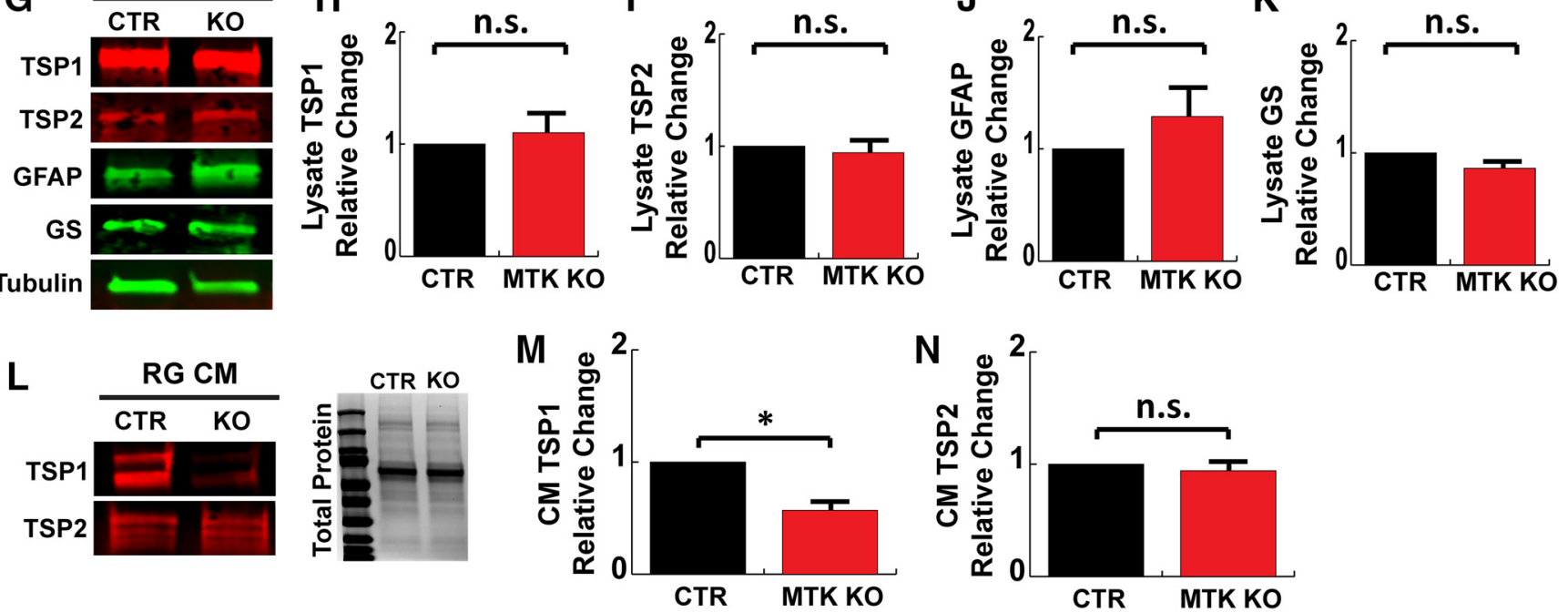

Figure 14. KO of MERTK in cultured retina glia (i.e., MG and retinal astrocytes) impairs synaptogenic function. $\boldsymbol{A}$, Schematic representation of coculture experiments. Purified RGCS were either cultured alone or cocultured with retinal glia. The retinal glia were plated onto transwell inserts, which were pretreated with CRISPR/SaCas9-AAVs $5 \mathrm{~d}$ before coculture with neurons (RGCs). For UCM treatment experiments, the control and MERTK KO retinal glia inserts were further treated with UTC conditioned medium prepared from cells transduced by lentiviruses containing control scramble shRNA (CTR UCM) or shRNAs against TSP1, TSP2, and TSP4 (TSP KD UCM) for 3 d before coculture with RGCs. $\boldsymbol{B}$, Schematic representation of the experimental design. Purified RGCS were treated with either control (SaCas9 only) or MERTK KO (MTK KO) retinal glia conditioned medium (RG CM). After 6 d treatment with conditioned media, the number of synapses is determined by immunocytochemistry. C, Representative images of RGCs stained with antibodies against presynaptic Bassoon (green, pre) and postsynaptic Homer (red, postsynaptic). White arrows indicate the colocalized synaptic puncta. Quantification of fold increase in synapse number of the RGCs that were either (D) cocultured or (E) treated with conditioned medium demonstrates that loss of MERTK in retina glia impairs their synaptogenic activity ( $n=30$ cells/condition). Data are mean \pm SEM. ${ }^{* * *} p<0.0001$. $F$, Treatment of MERTK KO retina glia with either scrambled shRNA transduced UCM or TSP1 + TSP2 + TSP4 knockdown shRNA transduced UTC conditioned medium (TSP-KD UCM) rescues the synaptogenic activity ( $n=30$ cells $/$ condition). Data are mean \pm SEM. ${ }^{* * *} p<0.0001,{ }^{*} p<0.05$. $\mathbf{G}$, Western blot analyses using the cell lysates from control and MERTK KO retina glia. Represented Western blot images are from an individual experiment with three biological replicates. Quantitative analysis of $(\boldsymbol{H})$ TSP1, (I) TSP2, ( $)$ GFAP, and $(\boldsymbol{K})$ GS ( $n=3$ per condition). $\boldsymbol{L}$, Left, Western blot analyses using the retina glia conditioned medium. Right, Total protein loaded is visualized by Coomassie blue staining. Quantitative analyses of retina glia-secreted $(\boldsymbol{M})$ TSP1 and $(\boldsymbol{N})$ TSP2 ( $n=3$ per condition). Data are mean \pm SEM. ${ }^{*} p<0.05$. 
washed with DPBS before coculture with RGCs to prevent direct interaction of the factors within the UCM and the RGCs. Quantification of the number of synapses in cultured RGCs revealed that pretreating MERTK KO retina glia with UCM rescued impaired synaptogenic function of these glial cells, as demonstrated by significantly greater number of synapses after coculture (Fig. $14 F$; one-way ANOVA, $\left.F_{(4,145)}=8.8020, p<0.0001\right)$. Triple knockdown of TSP family proteins from the hUTC did not completely inhibit this rescue effect of UCM, suggesting that TSP family proteins are not the only constituents of the hUTC-MG signaling that is involved in the rescue of the glial synaptogenic signaling.

Why do MERTK-KO retinal glia lose their synaptogenic function? To determine whether deletion of MERTK affects retinal gliasecreted synaptogenic TSPs, we examined the expression level of TSPs from the cell lysates and conditioned medium of CRISPR/ SaCas9 AAV-transduced cells. Quantitative analyses of the protein abundance indicated that the total amount of synaptogenic proteins TSP 1 and TSP 2 or glial markers GS and GFAP in the cell lysates were unchanged between control and MERTK-KO conditions (Fig. 14H-K). However, the amount of secreted TSP1 was significantly reduced in the retinal glia CM (Fig. 14M; Student's $t$ test, $p=0.0115$ ), whereas the TSP2 abundance was not affected (Fig. 14N; Student's $t$ test, $p=0.8002$ ). These results suggest that KO of MERTK in retinal glia affects TSP1 secretion and changes the composition of the glial-secretome that would impact many different aspects of retinal synaptogenesis and health.

\section{Discussion}

Our results provide novel insights into the mechanisms by which subretinal hUTC transplantation provides therapeutic effects in retinal degenerative diseases. Here we show that subretinal delivery of hUTC to RCS rats at P21, before PR loss, is critical to protect visual function, and repeating cell administration on P60 enhances the therapeutic benefits. We also demonstrate that synaptic impairments alongside morphological and functional changes in MG occur before the onset of significant PR loss in RCS rat retinas. hUTC transplantation protects synaptic connectivity and attenuates MG reactivity. In particular, MG-secreted synaptogenic factors TSP1 and TSP2 and their neuronal receptor $\alpha 2 \delta$ - 1 are sharply reduced, indicating an important role for diminished synaptogenic TSP signaling in the pathophysiology of the retinal degeneration observed in RCS rats. Interestingly, rescue of retinal function by hUTC transplantation strongly correlates with the protection of $\alpha 2 \delta$-1-containing synapses and attenuation of MG activation. CRISPR/SaCas9-mediated deletion of MERTK specifically in retinal glial cells was sufficient to recapitulate the reactive changes seen in the MG of RCS retinas. These reactive changes were also accompanied by reduced synaptic development providing a strong link between MG dysfunction and synaptic pathologies observed in RCS retinas. Furthermore, our in vitro experiments provide evidence that MERTK mutation has cell autonomous effects in MG, causing them to be less synaptogenic, potentially by disrupting TSP1 secretion. Importantly, secreted factors from hUTC restore the synaptogenic functions of retinal glia. Together, our findings reveal that the protective paracrine signals provided by hUTC improve the retinal microenvironment and synaptic connectivity at least in part through supporting MG function. Here we also provide the first evidence that MERTK mutation affects retinal health, not solely through the role of this protein in RPE. MG dysfunction due to loss of MERTK function in these glial cells may contribute significantly to loss of vision. Thus, our findings provide novel cellular mechanisms of action for hUTC in treating retinal degeneration and pinpoint $\mathrm{MG}$ as important cellular targets for therapy.

\section{Subretinal hUTC transplantation at P21 protects visual function and PRs}

Previous studies using the RCS rats have tested therapeutic efficacy of various cell sources, including hUTC (Lund et al., 2007), RPE (Wang et al., 2008), Schwann cells (McGill et al., 2007), retinal progenitor cells (Qu et al., 2015), and stem cell-derived RPE (Thomas et al., 2016). These studies showed that injection of cell therapy products between P21 and P28 could rescue visual function. Each of these studies administered their cell therapy of interest between P21 and P28, whereas only one study (Wang et al., 2008) compared the efficacy of RPE transplantation with a later time at P60. Similar to our findings, injection of RPE cells at P21 provides a superior therapeutic effect than administration at P60 (Wang et al., 2008). Even though it is not clear whether cell transplantation of another cell type on P60 could have potential beneficial effects, our results clearly show that subretinal transplantation of hUTC on P21, before significant PR death, is necessary for positive functional outcomes (Figs. 1,2). Visual acuity analysis and electrophysiological outcomes revealed that a repeated cell injection at P60 (in addition to P21) further enhances the rescue effect. This result indicates that the paracrine effects of hUTC last much longer than the cells; however, they diminish over time. Thus, repeated administration may present important benefits.

\section{Impaired synaptic development, TSP signaling, and MG reactivity precede PR loss in RCS rats}

The RCS rat contains a deletion mutation in the MERTK gene. It is widely accepted that MERTK deletion, which causes retinal degeneration in RCS rats, primarily affects phagocytosis of the PR outer segment debris by RPE cells (D'Cruz et al., 2000; Nandrot et al., 2000). MERTK is also expressed by microglia and MG in the retina (Roesch et al., 2008, 2012; Siegert et al., 2012). Importantly, the expression level of MERTK in MG significantly increases with degenerative stress (Roesch et al., 2012). Thus, the loss of this gene could have important effects on retinal function via multiple cell types and cellular mechanisms. In this study, we found that MERTK loss in MG is sufficient to induce reactive changes in these cells. These reactive changes are accompanied by reduced expression of GS. Furthermore, excitatory synapses formed in the OPL and IPL are severely affected, indicating that the ability of MG to support synapse development and maintenance is impaired. These results mimic the early MG-reactive changes and synaptic development deficits of the RCS rats that precede PR (PR) death (Fig. 2) and overt RPE dysfunction (Feng et al., 2002). These results show that loss of MERTK not only causes RPE dysfunction but also impairs MG contributing to important disease phenotypes. Interestingly, we did not find any evidence of PR loss in the MERTK KO retinas at P45, the latest time point we studied (data not shown). These combined results suggest that degeneration of PRs is mainly driven by loss of MERTK in other retinal cells, such as RPE, as previously reported (D'Cruz et al., 2000; Nandrot et al., 2000), whereas the synaptic development and connectivity deficits in the RCS rats are primarily driven by the MG dysfunction.

Previous studies demonstrated that astrocyte-secreted TSP1 and TSP2, which signal through their neuronal receptor $\alpha 2 \delta$-1, are necessary and sufficient for synapse formation in the brain (Christopherson et al., 2005; Eroglu et al., 2009; Risher and Eroglu, 2012). However, whether these proteins play important roles 
in retinal connectivity is not known. MG were previously proposed as a source of TSP1 in vitro and in vivo (Eichler et al., 2004; Yafai et al., 2014). Our study further extends these observations; and importantly, our findings put forth that impaired TSP $/ \alpha 2 \delta$ - 1 signaling is an underlying molecular mechanism in retinal degenerative diseases. In parallel with the reactive changes that occur in MG during early development (P14-P30), the expressions of TSP1, TSP2, and $\alpha 2 \delta$ - 1 are sharply decreased in the RCS rat retinas (Figs. $5-7)$. The reduced TSP $/ \alpha 2 \delta$-1 signaling strongly correlates with severely impaired synaptic development in both synaptic layers, OPL and IPL (Figs. 3, 4). The deficits in synaptic development are found before the onset of PR degeneration, and both synaptic layers are affected, suggesting that the failure in retinal synapse formation is caused by dysfunctional MG-driven synaptogenesis rather than PR loss.

\section{Subretinal hUTC transplantation attenuates reactive changes in MG and protects $\boldsymbol{\alpha} \mathbf{2} \delta$-1-containing synapses}

MG play a crucial role in modulating retinal function by providing physiological support to retinal neurons and regulating synaptic connectivity (Vecino et al., 2016). Thus, it is critical that MG function is preserved to maintain normal visual function. Dysfunctional MG are a common feature found in many animal models of retinal degeneration, including the RCS rat (Härtig et al., 1995; Fletcher and Kalloniatis, 1997; Dyer and Cepko, 2000; Zhao et al., 2010; Fernández-Sánchez et al., 2015; Hippert et al., 2015). The reactive changes of MG are characterized by morphological changes, including the loss of the "honeycomb"-like ensheathment surrounding PRs, process extension toward the subretinal space, and increased numbers of cell bodies as a result of MG proliferation (Dyer and Cepko, 2000; Fernández-Sánchez et al., 2015). Expression of GFAP is increased while GS expression is downregulated (Härtig et al., 1995). In our study, we show that early morphological changes indicating $M G$ activation precede PR loss. Remarkably, subretinal hUTC transplantation attenuates the reactive changes in the RCS rat retina. Our in vitro studies further demonstrate that retinal glia secrete synaptogenic factors, including TSP1 and TSP2, that induce synapse formation in RGCs. Ablating MERTK abolished retinal glia-induced synapse formation, and treatment of MERTK KO retina glia with UCM was sufficient to rescue the synaptogenic function. These results indicate that hUTC-secreted factors are sufficient to rescue the synaptogenic functions of MG. In parallel, previous studies have shown that hUTC secrete receptor tyrosine kinase ligands and rescue phagocytic defect in the of the RPE cells in RCS rats (Alder et al., 2012; Cao et al., 2016). These combined results strongly suggest that the therapeutic effects of subretinal hUTC transplantation are mediated by improving MG and RPE function, thus nurturing a healthy physiological microenvironment within the retina.

We found that $\alpha 2 \delta$-1-containing synapses are greatly reduced in RCS rats and are specifically preserved after hUTC transplantation. Furthermore, MERTK deletion in retinal glia decreases TSP1 secretion. Together, these results strongly indicate the importance of maintaining TSP $/ \alpha 2 \delta$-1-mediated synaptogenic signaling in preserving normal visual function. Our results also suggest that, even though hUTC themselves secrete TSPs, they preserve retinal synaptic connectivity primarily by improving MG-secretion of TSP1.

In conclusion, our findings reveal novel synaptic and glial mechanisms of subretinal hUTC transplantation for retinal degeneration. These findings indicate that hUTC provide signals that support PR health and MG function, and protects subsets of retinal synapses that are critical for maintaining vision. Further- more, our findings suggest that TSP $/ \alpha 2 \delta$ - 1 signaling is important for retinal function and is impaired in RCS retinas. These findings highlight MG and TSP $/ \alpha 2 \delta$-1 signaling as cellular and molecular targets for curing retinal degenerative diseases.

\section{References}

Alder J, Kramer BC, Hoskin C, Thakker-Varia S (2012) Brain-derived neurotrophic factor produced by human umbilical tissue-derived cells is required for its effect on hippocampal dendritic differentiation. Dev Neurobiol 72:755-765. CrossRef Medline

Allen NJ, Bennett ML, Foo LC, Wang GX, Chakraborty C, Smith SJ, Barres BA (2012) Astrocyte glypicans 4 and 6 promote formation of excitatory synapses via GluA1 AMPA receptors. Nature 486:410-414. CrossRef Medline

Bird AC, Phillips RL, Hageman GS (2014) Geographic atrophy: a histopathological assessment. JAMA Ophthalmol 132:338-345. CrossRef Medline

Cao J, Murat C, An W, Yao X, Lee J, Santulli-Marotto S, Harris IR, Inana G (2016) Human umbilical tissue-derived cells rescue retinal pigment epithelium dysfunction in retinal degeneration. Stem Cells 34:367-379. CrossRef Medline

Christopherson KS, Ullian EM, Stokes CC, Mullowney CE, Hell JW, Agah A, Lawler J, Mosher DF, Bornstein P, Barres BA (2005) Thrombospondins are astrocyte-secreted proteins that promote CNS synaptogenesis. Cell 120:421-433. CrossRef Medline

Cuenca N, Pinilla I, Sauvé Y, Lund R (2005) Early changes in synaptic connectivity following progressive photoreceptor degeneration in RCS rats. Eur J Neurosci 22:1057-1072. CrossRef Medline

D’Cruz PM, Yasumura D, Weir J, Matthes MT, Abderrahim H, LaVail MM, Vollrath D (2000) Mutation of the receptor tyrosine kinase gene mertk in the retinal dystrophic RCS rat. Hum Mol Genet 9:645-651. CrossRef Medline

Dowling JE, Sidman RL (1962) Inherited retinal dystrophy in the rat. J Cell Biol 14:73-109. CrossRef Medline

Dyer MA, Cepko CL (2000) Control of Müller glial cell proliferation and activation following retinal injury. Nat Neurosci 3:873-880. CrossRef Medline

Eichler W, Yafai Y, Wiedemann P, Reichenbach A (2004) Angiogenesisrelated factors derived from retinal glial (Müller) cells in hypoxia. Neuroreport 15:1633-1637. CrossRef Medline

Eroglu C, Allen NJ, Susman MW, O’Rourke NA, Park CY, Ozkan E, Chakraborty C, Mulinyawe SB, Annis DS, Huberman AD, Green EM, Lawler J, Dolmetsch R, Garcia KC, Smith SJ, Luo ZD, Rosenthal A, Mosher DF, Barres BA (2009) Gabapentin receptor alpha2delta-1 is a neuronal thrombospondin receptor responsible for excitatory CNS synaptogenesis. Cell 139:380-392. CrossRef Medline

Feng W, Yasumura D, Matthes MT, LaVail MM, Vollrath D (2002) Mertk triggers uptake of photoreceptor outer segments during phagocytosis by cultured retinal pigment epithelial cells. J Biol Chem 277:17016-17022. CrossRef Medline

Fernández-Sánchez L, Lax P, Campello L, Pinilla I, Cuenca N (2015) Astrocytes and Müller cell alterations during retinal degeneration in a transgenic rat model of retinitis pigmentosa. Front Cell Neurosci 9:484. CrossRef Medline

Fletcher EL, Kalloniatis M (1997) Neurochemical development of the degenerating rat retina. J Comp Neurol 388:1-22. CrossRef Medline

Girman SV, Wang S, Lund RD (2005) Time course of deterioration of rod and cone function in RCS rat and the effects of subretinal cell grafting: a light- and dark-adaptation study. Vision Res 45:343-354. CrossRef Medline

Gründer T, Kohler K, Guenther E (2001) Alterations in NMDA receptor expression during retinal degeneration in the RCS rat. Vis Neurosci 18: 781-787. CrossRef Medline

Härtig W, Grosche J, Distler C, Grimm D, el-Hifnawi E, Reichenbach A (1995) Alterations of Müller (glial) cells in dystrophic retinae of RCS rats. J Neurocytol 24:507-517. CrossRef Medline

Hippert C, Graca AB, Barber AC, West EL, Smith AJ, Ali RR, Pearson RA (2015) Müller glia activation in response to inherited retinal degeneration is highly varied and disease-specific. PLoS One 10:e0120415. CrossRef Medline

Ho AC, Chang TS, Samuel M, Williamson P, Willenbucher RF, Malone T (2017) Experience with a subretinal cell-based therapy in patients with geographic atrophy secondary to age-related macular degeneration. Am J Ophthalmol 179:67-80. CrossRef Medline

Hoon M, Okawa H, Della Santina L, Wong RO (2014) Functional architec- 
ture of the retina: development and disease. Prog Retin Eye Res 42:44-84. CrossRef Medline

Huang J, Zhou L, Wang H, Luo J, Zeng L, Xiong K, Chen D (2013) Distribution of thrombospondins and their neuronal receptor alpha2deltal in the rat retina. Exp Eye Res 111:36-49. CrossRef Medline

Ippolito DM, Eroglu C (2010) Quantifying synapses: an immunocytochemistry-based assay to quantify synapse number. J Vis Exp 45:2270. CrossRef Medline

Jiang Q, Thiffault C, Kramer BC, Ding GL, Zhang L, Nejad-Davarani SP, Li L, Arbab AS, Lu M, Navia B, Victor SJ, Hong K, Li QJ, Wang SY, Li Y, Chopp M (2012) MRI detects brain reorganization after human umbilical tissuederived cells (hUTC) treatment of stroke in rat. PLoS One 7:e42845. CrossRef Medline

Kimura N, Nishikawa S, Tamai M (2000) Müller cells in developing rats with inherited retinal dystrophy. Tohoku J Exp Med 191:157-166. CrossRef Medline

Klassen H (2016) Stem cells in clinical trials for treatment of retinal degeneration. Expert Opin Biol Ther 16:7-14. CrossRef Medline

Klimczak RR, Koerber JT, Dalkara D, Flannery JG, Schaffer DV (2009) A novel adeno-associated viral variant for efficient and selective intravitreal transduction of rat Müller cells. PLoS One 4:e7467. CrossRef Medline

Koh S, Kim N, Yin HH, Harris IR, Dejneka NS, Eroglu C (2015) Human umbilical tissue-derived cells promote synapse formation and neurite outgrowth via thrombospondin family proteins. J Neurosci 35:1564915665. CrossRef Medline

Kucukdereli H, Allen NJ, Lee AT, Feng A, Ozlu MI, Conatser LM, Chakraborty C, Workman G, Weaver M, Sage EH, Barres BA, Eroglu C (2011) Control of excitatory CNS synaptogenesis by astrocyte-secreted proteins hevin and SPARC. Proc Natl Acad Sci U S A 108:E440-E449. CrossRef Medline

Lim LS, Mitchell P, Seddon JM, Holz FG, Wong TY (2012) Age-related macular degeneration. Lancet 379:1728-1738. CrossRef Medline

Lund RD, Wang S, Lu B, Girman S, Holmes T, Sauvé Y, Messina DJ, Harris IR, Kihm AJ, Harmon AM, Chin FY, Gosiewska A, Mistry SK (2007) Cells isolated from umbilical cord tissue rescue photoreceptors and visual functions in a rodent model of retinal disease. Stem Cells 25:602-611. CrossRef Medline

McCarthy KD, de Vellis J (1980) Preparation of separate astroglial and oligodendroglial cell cultures from rat cerebral tissue. J Cell Biol 85:890-902. CrossRef Medline

McGill TJ, Lund RD, Douglas RM, Wang S, Lu B, Silver BD, Secretan MR, Arthur JN, Prusky GT (2007) Syngeneic Schwann cell transplantation preserves vision in RCS rat without immunosuppression. Invest Ophthalmol Vis Sci 48:1906-1912. CrossRef Medline

Moore TL, Pessina MA, Finklestein SP, Kramer BC, Killiany RJ, Rosene DL (2013) Recovery of fine motor performance after ischemic damage to motor cortex is facilitated by cell therapy in the rhesus monkey. Somatosens Mot Res 30:185-196. CrossRef Medline

Nandrot E, Dufour EM, Provost AC, Péquignot MO, Bonnel S, Gogat K, Marchant D, Rouillac C, Sépulchre de Condé B, Bihoreau MT, Shaver C, Dufier JL, Marsac C, Lathrop M, Menasche M, Abitbol MM (2000) Homozygous deletion in the coding sequence of the c-mer gene in RCS rats unravels general mechanisms of physiological cell adhesion and apoptosis. Neurobiol Dis 7:586-599. CrossRef Medline

Peng YW, Senda T, Hao Y, Matsuno K, Wong F (2003) Ectopic synaptogenesis during retinal degeneration in the royal college of surgeons rat. Neuroscience 119:813-820. CrossRef Medline

Prusky GT, Alam NM, Beekman S, Douglas RM (2004) Rapid quantification of adult and developing mouse spatial vision using a virtual optomotor system. Invest Ophthalmol Vis Sci 45:4611-4616. CrossRef Medline

Qu Z, Guan Y, Cui L, Song J, Gu J, Zhao H, Xu L, Lu L, Jin Y, Xu GT (2015) Transplantation of rat embryonic stem cell-derived retinal progenitor cells preserves the retinal structure and function in rat retinal degeneration. Stem Cell Res Ther 6:219. CrossRef Medline

Ran FA, Cong L, Yan WX, Scott DA, Gootenberg JS, Kriz AJ, Zetsche B, Shalem O, Wu X, Makarova KS, Koonin EV, Sharp PA, Zhang F (2015) In vivo genome editing using staphylococcus aureus Cas9. Nature 520: 186-191. CrossRef Medline

Risher WC, Eroglu C (2012) Thrombospondins as key regulators of synaptogenesis in the central nervous system. Matrix Biol 31:170-177. CrossRef Medline

Risher WC, Patel S, Kim IH, Uezu A, Bhagat S, Wilton DK, Pilaz LJ, Singh Alvarado J, Calhan OY, Silver DL, Stevens B, Calakos N, Soderling SH,
Eroglu C (2014) Astrocytes refine cortical connectivity at dendritic spines. eLife 3:04047. CrossRef Medline

Roesch K, Jadhav AP, Trimarchi JM, Stadler MB, Roska B, Sun BB, Cepko CL (2008) The transcriptome of retinal Müller glial cells. J Comp Neurol 509:225-238. CrossRef Medline

Roesch K, Stadler MB, Cepko CL (2012) Gene expression changes within Müller glial cells in retinitis pigmentosa. Mol Vis 18:1197-1214. Medline Siegert S, Cabuy E, Scherf BG, Kohler H, Panda S, Le YZ, Fehling HJ, Gaidatzis D, Stadler MB, Roska B (2012) Transcriptional code and disease map for adult retinal cell types. Nat Neurosci 15:487-495.S1-S2. CrossRef Medline

Singh SK, Stogsdill JA, Pulimood NS, Dingsdale H, Kim YH, Pilaz LJ, Kim IH, Manhaes AC, Rodrigues WS Jr, Pamukcu A, Enustun E, Ertuz Z, Scheiffele P, Soderling SH, Silver DL, Ji RR, Medina AE, Eroglu C (2016) Astrocytes assemble thalamocortical synapses by bridging NRX1alpha and NL1 via hevin. Cell 164:183-196. CrossRef Medline

Thomas BB, Zhu D, Zhang L, Thomas PB, Hu Y, Nazari H, Stefanini F, Falabella P, Clegg DO, Hinton DR, Humayun MS (2016) Survival and functionality of hESC-derived retinal pigment epithelium cells cultured as a monolayer on polymer substrates transplanted in RCS rats. Invest Ophthalmol Vis Sci 57:2877-2887. CrossRef Medline

Trachtenberg MC, Packey DJ (1983) Rapid isolation of mammalian Müller cells. Brain Res 261:43-52. CrossRef Medline

Tso MO, Zhang C, Abler AS, Chang CJ, Wong F, Chang GQ, Lam TT (1994) Apoptosis leads to photoreceptor degeneration in inherited retinal dystrophy of RCS rats. Invest Ophthalmol Vis Sci 35:2693-2699. Medline

Ullian EM, Sapperstein SK, Christopherson KS, Barres BA (2001) Control of synapse number by glia. Science 291:657-661. CrossRef Medline

Vecino E, Rodriguez FD, Ruzafa N, Pereiro X, Sharma SC (2016) Glianeuron interactions in the mammalian retina. Prog Ret Eye Res 51:1-40. CrossRef Medline

Venugopalan P, Wang Y, Nguyen T, Huang A, Müller KJ, Goldberg JL (2016) Transplanted neurons integrate into adult retinas and respond to light. Nat Commun 7:10472. CrossRef Medline

Wang S, Lu B, Lund RD (2005) Morphological changes in the Royal College of Surgeons rat retina during photoreceptor degeneration and after cellbased therapy. J Comp Neurol 491:400-417. CrossRef Medline

Wang S, Lu B, Girman S, Holmes T, Bischoff N, Lund RD (2008) Morphological and functional rescue in RCS rats after RPE cell line transplantation at a later stage of degeneration. Invest Ophthalmol Vis Sci 49:416-421. CrossRef Medline

Werblin FS, Dowling JE (1969) Organization of the retina of the mudpuppy, Necturus maculosus: II. Intracellular recording. J Neurophysiol 32: 339-355. CrossRef Medline

Williams PR, Suzuki SC, Yoshimatsu T, Lawrence OT, Waldron SJ, Parsons MJ, Nonet ML, Wong RO (2010) In vivo development of outer retinal synapses in the absence of glial contact. J Neurosci 30:11951-11961. CrossRef Medline

Winzeler A, Wang JT (2013) Purification and culture of retinal ganglion cells from rodents. Cold Spring Harbor Protoc 2013:643-652. CrossRef Medline

Yafai Y, Eichler W, Iandiev I, Unterlauft JD, Jochmann C, Wiedemann P, Bringmann A (2014) Thrombospondin-1 is produced by retinal glial cells and inhibits the growth of vascular endothelial cells. Ophthalmic Res 52:81-88. CrossRef Medline

Zhang L, Li Y, Zhang C, Chopp M, Gosiewska A, Hong K (2011) Delayed administration of human umbilical tissue-derived cells improved neurological functional recovery in a rodent model of focal ischemia. Stroke 42:1437-1444. CrossRef Medline

Zhang L, Li Y, Romanko M, Kramer BC, Gosiewska A, Chopp M, Hong K (2012) Different routes of administration of human umbilical tissuederived cells improve functional recovery in the rat after focal cerebral ischemia. Brain Res 1489:104-112. CrossRef Medline

Zhang L, Yi L, Chopp M, Kramer BC, Romanko M, Gosiewska A, Hong K (2013) Intravenous administration of human umbilical tissue-derived cells improves neurological function in aged rats after embolic stroke. Cell Transplant 22:1569-1576. CrossRef Medline

Zhao C, Wang Q, Temple S (2017) Stem cell therapies for retinal diseases: recapitulating development to replace degenerated cells. Development 144:1368-1381. CrossRef Medline

Zhao TT, Tian CY, Yin ZQ (2010) Activation of Müller cells occurs during retinal degeneration in RCS rats. Adv Exp Med Biol 664:575-583. CrossRef Medline 